QUARTERLY OF APPLIED MATHEMATICS

VOLUME LXVI, NUMBER 2

JUNE 2008, PAGES 325-350

S 0033-569X(08)01106-7

Article electronically published on March 12, 2008

\title{
AN EVOLUTIONARY WEIGHTED $p$-LAPLACIAN WITH NEUMANN BOUNDARY VALUE CONDITION IN A PERFORATED DOMAIN
}

\author{
BY \\ YUANYUAN KE (Department of Mathematics, Jilin University, Changchun, Jilin 130012, People's \\ Republic of China) (School of Mathematics \& Computational Science, Sun Yat-Sen University, \\ Guangzhou 510275, People's Republic of China), \\ JINGXUE YIN (Department of Mathematics, Jilin University, Changchun, Jilin 130012, People's \\ Republic of China), \\ AND \\ CHUNHUA JIN (Department of Mathematics, Jilin University, Changchun, Jilin 130012, People's \\ Republic of China)
}

Abstract. In this paper, we study an evolutionary weighted $p$-Laplacian with Neumann boundary value condition in a perforated domain. We discuss the removability of the orifice for the radially symmetric steady solution, the general steady solution and for the evolutionary solution of the problem considered.

1. Introduction. Let $\Omega \subset \mathbb{R}^{n}$ be a bounded domain with smooth boundary, $0 \in \Omega$. We consider the following problem in the perforated domain $\Omega \backslash\{0\}$ :

$$
\begin{gathered}
\frac{\partial u}{\partial t}-\operatorname{div}\left(|x|^{\alpha}|\nabla u|^{p-2} \nabla u\right)=f(x, t), \quad(x, t) \in R_{T}, \\
u(x, 0)=u_{0}(x), \quad x \in \Omega \backslash\{0\}, \\
|x|^{\alpha}|\nabla u|^{p-2} \nabla u \cdot \vec{\nu}=g(x, t), \quad(x, t) \in \partial \Omega \times(0, T),
\end{gathered}
$$

where $R_{T}=(\Omega \backslash\{0\}) \times(0, T), \Omega \backslash\{0\}$ can be considered as the limit of $\Omega \backslash B_{\varepsilon}, B_{\varepsilon}$ is a ball with radius $\varepsilon$ small enough, $\alpha>0, p>1, n \geq 1, \vec{\nu}$ denotes the unit outward

Received July 13, 2006.

2000 Mathematics Subject Classification. 35D05, 35B05, 35B45, 35B40.

Key words and phrases. Evolutionary weighted $p$-Laplacian, perforated domain, orifice.

This work is partially supported by NNSF of China, partially supported by NSFGD-06300481, partially supported by a Specific Foundation for Ph.D. Specialities of Educational Department of China, and partially supported by 985 Projects.

Corresponding author. email: jinchhua@126.com.

E-mail address: keyy@jlu.edu.cn

E-mail address: yjx@jlu.edu.cn

E-mail address: jinchhua@126.com 
normal to the boundary $\partial \Omega$, and $f, g$ and $u_{0}$ are all bounded functions satisfying some compatibility conditions. Such a problem originates from many physical backgrounds, for example non-Newtonian fluids, and has, in general, degeneracy and singularity; see [1- 4.

Assume that $u$, which is appropriately smooth, is a solution of the equation (1.1) in $\Omega \backslash\{0\}$ satisfying the conditions (1.2) and (1.3). The main subject of this paper is to study the asymptotic behavior of the solution $u$ towards the origin. Exactly speaking, this paper aims to solve the following two problems. The first one is whether the limit $\lim _{x \rightarrow 0} u(x, t)$ exists, and if the limit exists, whether it can be determined uniquely by the initial and boundary value conditions (1.2), (1.3). In the case of nonuniqueness, can we prescribe the value of $u(0, t)$, namely

$$
u(0, t)=q(t),
$$

so that the problem (1.1)-(1.4) always has a solution? The second one is under what conditions a solution $u$ of the problem in the perforated domain $\Omega \backslash\{0\}$ is also the solution of the corresponding problems in the domain $\Omega$ without an orifice. As for the second question, we note that on the one hand, exerting some proper boundary value conditions at the orifice, the solutions of the problem in the perforated domain can also solve the problem in the domain without an orifice. On the other hand, more importantly, there may be such a situation, in which whatever boundary value conditions at the orifice are exerted, all the solutions of the problem in the perforated domain cannot solve the problem in the domain without an orifice. That means, there are two possibilities to the orifice, removable and unremovable. The so-called unremovable orifice means that whatever boundary value condition is exerted on it, the solution of the problem in the perforated domain cannot solve the problem in the domain without an orifice. Otherwise, we say the orifice is removable.

There is a rich literature concerning partial differential equations in perforated domains; see [5]-12. The main characteristics of these problems are that all the problems are studied on such a domain which is the limit of the remaining domain after digging out one or several small balls from it. The main interest lies in the study of the asymptotic behavior of solutions when the radii of the small balls shrink to one point. From those works, see [13]- 17], a very important method in studying the properties of the solutions is firstly to discuss the radially symmetric steady solutions, which can be regarded as a special class of the problems in perforated domains.

Problems which are studied in a perforated domain $\Omega \backslash\{0\}$ are obviously different from the problems in the domain $\Omega$ without an orifice, while they also have a close relationship. It is certain that the solution of the problem in the domain $\Omega$ without an orifice is always the solution of the problem in the corresponding perforated domain, but the contrary might not be true. As we know, if the orifice is removable, one can obtain some special characteristics of the solution by studying its radially symmetric steady solutions. On the other hand, if the orifice is unremovable, it is also much more interesting to analyze the singular properties of solutions of the problem. Therefore, it is important to discuss the properties of solutions near the orifice. 
In this paper, we first discuss the radially symmetric steady solutions. By analyzing the results of the radially symmetric steady problem, we gain the existence and uniqueness of solutions and present the accurate condition whether the orifice is unremovable or removable. We also discuss the general steady solutions and the evolutionary solutions separately. We use a series of methods, such as doing the a priori estimates in a weighted space, establishing the comparison principle, etc., to gain the existence and uniqueness of the solutions for the problem. Although we can obtain the conditions whether the orifice is unremovable or removable in the case of radially symmetric steady states, we encounter some difficulties when facing the general steady states and the evolutionary case. Finally, when dealing with the above-listed difficulties, we only use the sup-solutions and sub-solutions to show that under some circumstances the origin is unremovable.

This paper is organized in the following way. Section 2 contains the discussion of the radially symmetric steady solution. Section 3 is devoted to studying the general steady solution. Finally in Section 4, the evolutionary solution is considered.

2. Radially symmetric steady solution. This section is devoted to a special class of solutions, namely the radially symmetric steady solution.

Let $\Omega$ be the unit ball $B, f(x)$ and $g(x)$ be radially symmetric, namely, $f(x)=f(|x|)$ and $g(x)=g(|x|)$. Then the problem (1.1), (1.3), (1.4) can be rewritten as

$$
\begin{gathered}
-\operatorname{div}\left(|x|^{\alpha}|\nabla u|^{p-2} \nabla u\right)=f(|x|), \quad x \in B \backslash\{0\}, \\
|x|^{\alpha}|\nabla u|^{p-2} \nabla u \cdot \vec{\nu}=A, \quad x \in \partial B, \\
\lim _{x \rightarrow 0} u(|x|)=\theta,
\end{gathered}
$$

where $A=g(|x|)$ for $x$ on $\partial B, \theta$ is a constant, and the problem in the domain without an orifice is

$$
-\operatorname{div}\left(|x|^{\alpha}|\nabla u|^{p-2} \nabla u\right)=f(|x|), \quad x \in B,
$$

with boundary value condition (2.2).

Definition 2.1. A function $u$ is said to be a solution of the problem (2.4), (2.2) if $u \in C\left(\overline{B_{1}}\right) \cap W^{1, p}\left(B_{1}\right),|x|^{\alpha}|\nabla u|^{p} \in L^{1}\left(B_{1}\right)$, and $u$ satisfies

$$
\int_{B_{1}}|x|^{\alpha}|\nabla u|^{p-2} \nabla u \nabla \varphi d x-\int_{\partial B_{1}} A \varphi d \sigma=\int_{B_{1}} f \varphi d x
$$

for any $\varphi \in C^{\infty}\left(\overline{B_{1}}\right)$, where $\nabla u$ is the generalized gradient of $u$ in $B_{1}$.

Definition 2.2. A function $u$ is said to be a solution of the problem (2.1)-2.3) if $u \in C\left(\overline{B_{1}}\right), \forall 0<\delta<1, u \in W^{1, p}\left(B_{1} \backslash B_{\delta}\right),|x|^{\alpha}\left|\nabla^{*} u\right|^{p} \in L^{1}\left(B_{1}\right)$, and $u$ satisfies

$$
\int_{B_{1}}|x|^{\alpha}\left|\nabla^{*} u\right|^{p-2} \nabla^{*} u \nabla \varphi d x-\int_{\partial B_{1}} A \varphi d \sigma=\int_{B_{1}} f \varphi d x
$$

for any $\varphi \in C^{\infty}\left(\overline{B_{1}}\right)$ with $\operatorname{dist}\{\operatorname{supp} \varphi, 0\}>0$, where $\nabla^{*} u$ is the generalized gradient of $u$ in $B_{1} \backslash\{0\}$, and (2.3) holds.

But for simplicity, in the situation without confusion, we still use $\nabla u$ instead of $\nabla^{*} u$ later. 
2.1. Existence and uniqueness. We first consider the existence and uniqueness of the solutions for the problem (2.1)-(2.3).

Theorem 2.3. Assume $A+\int_{0}^{1} r^{n-1} f(r) d r \neq 0$ and $\alpha>0$. If $p>n+\alpha$, then there exists one and only one solution of the problem (2.1) - (2.3); while if $1<p \leq n+\alpha$, there is no solution of the problem (2.1)-(2.3).

Proof. By a direct calculation, we get the radially symmetric form of the problem (2.1) -2.3 :

$$
\begin{gathered}
-\left(r^{n-1+\alpha} \phi_{p}\left(u^{\prime}\right)\right)^{\prime}=r^{n-1} f(r), \quad r \in(0,1), \\
\phi_{p}\left(u^{\prime}(1)\right)=A, \\
\lim _{r \rightarrow 0} u(r)=\theta,
\end{gathered}
$$

where $\phi_{p}(s)=|s|^{p-2} s$. Integrating the equation (2.5) from $r$ to 1 , we have

$$
r^{n+\alpha-1} \phi_{p}\left(u^{\prime}(r)\right)=A+\int_{r}^{1} s^{n-1} f(s) d s .
$$

Hence

$$
\begin{aligned}
u^{\prime}(r) & =\phi_{q}\left(\frac{1}{r^{n+\alpha-1}}\left(A+\int_{r}^{1} s^{n-1} f(s) d s\right)\right) \\
& =\frac{1}{r^{\frac{n+\alpha-1}{p-1}}} \phi_{q}\left(A+\int_{r}^{1} s^{n-1} f(s) d s\right),
\end{aligned}
$$

where $\phi_{q}=\phi_{p}^{-1}, \phi_{q}(s)=|s|^{q-2} s$, and $1 / p+1 / q=1$. When $A+\int_{0}^{1} r^{n-1} f(r) d r \neq 0$ and $p>n+\alpha$, the above equation is integrable on $(0, r)$. Thanks to the condition (2.7), it follows that

$$
u(r)=\theta+\int_{0}^{r}\left(\frac{1}{\tau^{\frac{n+\alpha-1}{p-1}}} \phi_{q}\left(A+\int_{\tau}^{1} s^{n-1} f(s) d s\right)\right) d \tau .
$$

It can be proved that $u(|x|)$ is the solution of the problem (2.1)-(2.3). Therefore, there exist solutions of the problem (2.1)-(2.3). The proof of the uniqueness of the solutions is similar to that of Theorem 3.2, which can be seen later.

From the above we see that when $1<p \leq n+\alpha$, there is no solution of the problem (2.1) - (2.3). The proof is complete.

From the proof of Theorem 2.3 it is easy to see that

REMARK 2.4. When $A+\int_{0}^{1} s^{n-1} f(s) d s=C \neq 0$, for any $u(0)=\theta$, it follows that $\lim _{r \rightarrow 0} r^{n+\alpha-1} \phi_{p}\left(u^{\prime}\right) \neq 0$; while if $A+\int_{0}^{1} s^{n-1} f(s) d s=0$, for any $u(0)=\theta$, we have $\lim _{r \rightarrow 0} r^{n+\alpha-1} \phi_{p}\left(u^{\prime}\right)=0$.

Theorem 2.5. Assume $A+\int_{0}^{1} r^{n-1} f(r) d r=0$ and $\alpha>0$. If $p>\alpha$, there exists one and only one solution of the problem (2.1)-(2.3); while if $1<p \leq \alpha$, and $|f|>0$ in a neighborhood of 0 , then there is no solution of the problem (2.1)-(2.3). 
Proof. Case 1. $p>\alpha+1$. Considering the problem (2.5) (2.7), and applying the methods similar to Theorem 2.3 , we obtain that

$$
\begin{aligned}
u^{\prime}(r) & =\frac{1}{r^{\frac{n+\alpha-1}{p-1}}} \phi_{q}\left(A+\int_{r}^{1} s^{n-1} f(s) d s\right) \\
& =-\frac{1}{r^{\frac{n+\alpha-1}{p-1}}} \phi_{q}\left(\int_{0}^{r} s^{n-1} f(s) d s\right) .
\end{aligned}
$$

Since $f$ is bounded, it follows that

$$
C_{1} r^{\frac{1-\alpha}{p-1}} \leq u^{\prime}(r) \leq C_{2} r^{\frac{1-\alpha}{p-1}}
$$

where $C_{1}$ and $C_{2}$ both depend on the bound of $f$. Therefore, when $p>\alpha+1, u^{\prime}$ is integrable on $[0, r]$. Recalling the condition (2.7), we have

$$
u(r)=\theta-\int_{0}^{r}\left(\frac{1}{\tau^{\frac{n+\alpha-1}{p-1}}} \phi_{q}\left(\int_{0}^{\tau} s^{n-1} f(s) d s\right)\right) d \tau .
$$

One can verify that $u(|x|)$ satisfies the problem (2.1)-(2.3). The proof of the uniqueness of the solutions is similar to that of Theorem 3.2. which can be seen later.

Case 2. $\alpha<p \leq \alpha+1$. By utilizing a similar method to case 1 , we see that the problem (2.1)-(2.2) admits solutions. So, in what follows, we only need to show the uniqueness of solutions. Let

$$
F_{\varepsilon}=\{x \neq 0 ; \operatorname{dist}(x, 0)>\varepsilon\} .
$$

Set $\xi_{\varepsilon} \in C_{0}^{\infty}\left(F_{\varepsilon}\right)$ with $0 \leq \xi \leq 1 ; \xi_{\varepsilon}=1$, on $F_{2 \varepsilon} ; \xi_{\varepsilon}=0$, on $B \backslash F_{\varepsilon} ;\left|\nabla \xi_{\varepsilon}\right| \leq C / \varepsilon$, where $C$ is a constant independent of $\varepsilon$.

Let $u$ and $v$ be two solutions of the problem (2.1)-(2.2). Then for every $\varphi \in C^{\infty}(\bar{B})$ and $\operatorname{dist}\{\operatorname{supp} \varphi, 0\}>0$, it follows that

$$
\int_{B}|x|^{\alpha}\left(|\nabla u|^{p-2} \nabla u-|\nabla v|^{p-2} \nabla v\right) \nabla \varphi d x=0 .
$$

Setting $\varphi=(u-v) \xi_{\varepsilon}$, we have

$$
\begin{aligned}
& \int_{B}|x|^{\alpha}\left(|\nabla u|^{p-2} \nabla u-|\nabla v|^{p-2} \nabla v\right)(\nabla u-\nabla v) \xi_{\varepsilon} d x \\
& =-\int_{B}|x|^{\alpha}\left(|\nabla u|^{p-2} \nabla u-|\nabla v|^{p-2} \nabla v\right) \nabla \xi_{\varepsilon}(u-v) d x \\
& \leq \int_{B}|u-v||x|^{\alpha}\left(|\nabla u|^{p-1}+|\nabla v|^{p-1}\right)\left|\nabla \xi_{\varepsilon}\right| d x \\
& =\int_{F_{\varepsilon} \backslash F_{2 \varepsilon}}|u-v||x|^{\alpha}\left(|\nabla u|^{p-1}+|\nabla v|^{p-1}\right)\left|\nabla \xi_{\varepsilon}\right| d x \\
& \leq C\left(\int_{F_{\varepsilon} \backslash F_{2 \varepsilon}}|x|^{\alpha}\left(|\nabla u|^{p}+|\nabla v|^{p}\right)\right)^{\frac{p-1}{p}} \varepsilon^{\frac{\alpha+1-p}{p}} .
\end{aligned}
$$

Since $p \leq 1+\alpha$, letting $\varepsilon \rightarrow 0^{+}$, then

$$
C\left(\int_{F_{\varepsilon} \backslash F_{2 \varepsilon}}|x|^{\alpha}\left(|\nabla u|^{p}+|\nabla v|^{p}\right)\right)^{\frac{p-1}{p}} \varepsilon^{\frac{\alpha+1-p}{p}} \rightarrow 0,
$$


which implies that

$$
\int_{B}|x|^{\alpha}\left(|\nabla u|^{p-2} \nabla u-|\nabla v|^{p-2} \nabla v\right)(\nabla u-\nabla v) \xi_{\varepsilon} d x \rightarrow 0 \quad\left(\varepsilon \rightarrow 0^{+}\right) .
$$

Notice that

$$
|x|^{\alpha}\left(|\nabla u|^{p-2} \nabla u-|\nabla v|^{p-2} \nabla v\right)(\nabla u-\nabla v) \xi_{\varepsilon} \geq 0
$$

and $|x|^{\alpha}>0, \xi_{\varepsilon}=1$ for $x \in F_{2 \varepsilon}$. Then we have that $\nabla u=\nabla v$ a.e. in $B$. Combining with (2.3), we finally arrive at $u=v$ a.e. in $B$.

Case 3. $1<p \leq \alpha$. Recalling (2.9), and noticing that $|f|>0$ in a neighborhood of 0 , without loss of generality, we might as well assume that $f>0$ in a small neighborhood of 0 . Then there exist $c_{1}$ and $c_{2}$ with $c_{1}>c_{2}>0$ such that

$$
-c_{1} r^{\frac{1-\alpha}{p-1}} \leq u^{\prime}(r) \leq-c_{2} r^{\frac{1-\alpha}{p-1}}
$$

Clearly, no solution exists if $1<p \leq \alpha$.

2.2. Unremovable orifice. In this part, we discuss the properties of the orifice.

Theorem 2.6. If $A+\int_{0}^{1} r^{n-1} f(r) d r \neq 0, p>n+\alpha$ and $\alpha>0$, the origin is the unremovable orifice for the problem (2.1)-(2.3). While if $A+\int_{0}^{1} r^{n-1} f(r) d r=0$ and $0<\alpha<p$, the origin is the removable orifice for the problem (2.1) -2.3).

Proof. For the case $A+\int_{0}^{1} r^{n-1} f(r) d r \neq 0, p>n+\alpha$ and $\alpha>0$, we only need to prove that for all $\theta$, the solution of the problem (2.1)-2.3) is not the solution of the problem (2.4), (2.2). We consider the radially symmetric form of the problem. Since $A+\int_{0}^{1} s^{n-1} f(s) d s \neq 0$, it follows that

$$
\begin{aligned}
& \int_{0}^{1} r^{n-1+\alpha} \phi_{p}\left(u^{\prime}\right) \varphi^{\prime} d r-A \varphi(1) \\
& =\int_{0}^{1}\left(A+\int_{r}^{1} s^{n-1} f(s) d s\right) \varphi^{\prime} d r-A \varphi(1) \\
& =A \varphi(1)-\left(A+\int_{0}^{1} s^{n-1} f(s) d s\right) \varphi(0)+\int_{0}^{1} r^{n-1} f(r) \varphi d r-A \varphi(1) \\
& =\int_{0}^{1} r^{n-1} f(r) \varphi d r-\left(A+\int_{0}^{1} s^{n-1} f(s) d s\right) \varphi(0) \\
& \neq \int_{0}^{1} r^{n-1} f(r) \varphi d r
\end{aligned}
$$

where $\varphi \in C[0,1]$. The inequality implies that $u$ is not the solution of (2.4), (2.2).

For the case $A+\int_{0}^{1} r^{n-1} f(r) d r=0$ and $0<\alpha<p$, we only need to prove there exists $\theta$, such that the solution $u$ of the problem (2.1)-(2.3) also satisfies the problem (2.4), 
(2.2). For any $\varphi \in C[0,1]$, we have

$$
\begin{aligned}
& \int_{0}^{1} r^{n-1+\alpha} \phi_{p}\left(u^{\prime}\right) \varphi^{\prime} d r-A \varphi(1) \\
= & -\int_{0}^{1}\left(\int_{0}^{r} s^{n-1} f(s) d s\right) \varphi^{\prime} d r-A \varphi(1) \\
= & -\left(A+\int_{0}^{1} s^{n-1} f(s) d s\right) \varphi(1)+\int_{0}^{1} r^{n-1} f(r) \varphi d r \\
= & \int_{0}^{1} r^{n-1} f(r) \varphi d r .
\end{aligned}
$$

The proof is complete.

REMARK 2.7. By Theorem 2.6, we see that the condition $A+\int_{0}^{1} s^{n-1} f(s) d s \neq 0$, i.e. $\lim _{r \rightarrow 0} r^{n+\alpha-1} \phi_{p}\left(u^{\prime}(r)\right) \neq 0$, is the sufficient condition which guarantees that the origin is an unremovable orifice of the problem (2.1)-(2.3).

For the problem (2.5)-(2.7), when $A+\int_{0}^{1} r^{n-1} f(r) d r \neq 0$, we also get

Proposition 2.8. When $A+\int_{0}^{1} s^{n-1} f(s) d s \neq 0$ and $p>n+\alpha, \alpha>0$, we have

$$
u^{\prime}(r)=O^{\#}\left(\frac{1}{r^{\frac{n+\alpha-1}{p-1}}}\right), \quad r \rightarrow 0 .
$$

Proof. Falling back to (2.8), and since $A+\int_{0}^{1} s^{n-1} f(s) d s \neq 0$, we have

$$
\begin{aligned}
& \lim _{r \rightarrow 0} u^{\prime}(r) r^{\frac{n+\alpha-1}{p-1}}=\lim _{r \rightarrow 0} \phi_{q}\left(A+\int_{r}^{1} s^{n-1} f(s) d s\right) \\
& =\phi_{q}\left(A+\int_{0}^{1} s^{n-1} f(s) d s\right) \neq 0,
\end{aligned}
$$

which implies that when $r \rightarrow 0, u^{\prime}(r)$ is of the same order as $\frac{1}{r^{\frac{n+\alpha-1}{p-1}}}$. The proof is complete.

For the solution $u$ of the problem (2.5)-(2.7), if $A+\int_{0}^{1} s^{n-1} f(s) d s=0$, we have

Proposition 2.9. If $\alpha<1, u^{\prime}(0)=0$; if $\alpha=1, u^{\prime}(0)=C f(0)^{q-1}$; if $1<\alpha<p, u^{\prime}$ is integrable in $(0,1)$.

In fact, if $\alpha<1$, we have $(1-\alpha) /(p-1)>0$. Then $u^{\prime}(0)=0$, when $r \rightarrow 0$. If $\alpha=1$, we have $(1-\alpha) /(p-1)=0$. Then $u^{\prime}(0)=C f(0)^{q-1}$, when $r \rightarrow 0$. While if $\alpha>1$ and $\frac{\alpha-1}{p-1}<1$, i.e. if $1<\alpha<p$, from (2.9), we see that $u^{\prime}$ is integrable in $(0,1)$.

3. General steady solution. In this section, we consider the following problem:

$$
\begin{gathered}
-\operatorname{div}\left(|x|^{\alpha}|\nabla u|^{p-2} \nabla u\right)=f(x), \quad x \in \Omega \backslash\{0\}, \\
|x|^{\alpha}|\nabla u|^{p-2} \nabla u \cdot \vec{\nu}=g(x), \quad x \in \partial \Omega, \\
\lim _{x \rightarrow 0} u(x)=\theta
\end{gathered}
$$

in the perforated domain $\Omega \backslash\{0\}$. Here $f(x)$ and $g(x)$ satisfy

$$
h_{1}(|x|) \leq f(x) \leq h_{2}(|x|), \quad M_{1} \leq g(x) \leq M_{2},
$$


where $h_{1}$ and $h_{2}$ are radially symmetric and bounded functions, and $M_{1}$ and $M_{2}$ are both constants.

Definition 3.1. A function $u$ is said to be a solution of the problem (3.1)-(3.3) if $u \in C(\bar{\Omega})$, for every $\delta>0, u \in W^{1, p}\left(\Omega \backslash B_{\delta}\right),|x|^{\alpha}\left|\nabla^{*} u\right|^{p} \in L^{1}(\Omega)$, and $u$ satisfies

$$
\int_{\Omega}|x|^{\alpha}\left|\nabla^{*} u\right|^{p-2} \nabla^{*} u \nabla \varphi d x-\int_{\partial \Omega} g \varphi d \sigma=\int_{\Omega} f \varphi d x
$$

for any $\varphi \in C^{\infty}(\bar{\Omega})$ with $\operatorname{dist}\{\operatorname{supp} \varphi, 0\}>0$, where $\nabla^{*} u$ is the generalized gradient of $u$ in $\Omega \backslash\{0\}$, and (3.3) holds.

\subsection{Existence and uniqueness.}

TheOREM 3.2. When $p>n+\alpha$, there is one and only one solution of the problem (3.1) $-(3.3)$.

In order to study the existence of solutions in the perforated domain $\Omega \backslash\{0\}$, we should first consider an approximate problem in the domain $\Omega \backslash B_{\delta}$, where $\delta>0$ is a small positive constant, namely

$$
\begin{gathered}
\operatorname{div}\left(|x|^{\alpha}\left|\nabla u_{\delta}\right|^{p-2} \nabla u_{\delta}\right)=f(x), \quad x \in \Omega \backslash B_{\delta}, \\
|x|^{\alpha}\left|\nabla u_{\delta}\right|^{p-2} \nabla u_{\delta} \cdot \vec{\nu}=g(x), \quad x \in \partial \Omega, \\
u_{\delta}(x)=\theta, \quad x \in \partial B_{\delta} .
\end{gathered}
$$

We call $u_{\delta}$ the solution of the problem (3.4)-(3.6) if $u_{\delta} \in C^{\beta}\left(\overline{\Omega \backslash B_{\delta}}\right) \cap W^{1, p}\left(\Omega \backslash B_{\delta}\right)$, $|x|^{\alpha}\left|\nabla u_{\delta}\right|^{p} \in L^{1}\left(\Omega \backslash B_{\delta}\right)$, and $u$ satisfies

$$
\int_{\Omega \backslash B_{\delta}}|x|^{\alpha}\left|\nabla u_{\delta}\right|^{p-2} \nabla u_{\delta} \nabla \varphi d x-\int_{\partial \Omega} g \varphi d \sigma=\int_{\Omega \backslash B_{\delta}} f \varphi d x,
$$

for any $\varphi \in C^{\infty}\left(\overline{\Omega \backslash B_{\delta}}\right)$, dist $\left\{\operatorname{supp} \varphi, \partial B_{\delta}\right\}>0,0<\beta<1$, and (3.6) holds.

Lemma 3.3. There exists one and only one solution of the problem (3.4)-(3.6).

Proof. For the degeneracy of the equation (3.4), we firstly consider the homogeneous problem

$$
\begin{gathered}
-\operatorname{div}\left(|x|^{\alpha}\left(\left|\nabla u_{\varepsilon \delta}\right|^{2}+\varepsilon\right)^{\frac{p-2}{2}} \nabla u_{\varepsilon \delta}\right)=f(x), \quad x \in \Omega \backslash B_{\delta}, \\
|x|^{\alpha}\left(\left|\nabla u_{\varepsilon \delta}\right|^{2}+\varepsilon\right)^{\frac{p-2}{2}} \nabla u_{\varepsilon \delta} \cdot \vec{\nu}=g(x), \quad x \in \partial \Omega, \\
u_{\varepsilon \delta}(x)=\theta, \quad x \in \partial B_{\delta},
\end{gathered}
$$

where $\varepsilon>0$. By the classical theories of the elliptic equations (see for example [18), the problem (3.8)-(3.10) admits a unique solution $u_{\varepsilon \delta}$ with $u_{\varepsilon \delta} \in C^{\beta}\left(\overline{\Omega \backslash B_{\delta}}\right) \cap W^{1, p}\left(\Omega \backslash B_{\delta}\right)$ and $|x|^{\alpha}\left|\nabla u_{\varepsilon \delta}\right|^{p} \in L^{1}\left(\Omega \backslash B_{\delta}\right)$. Here the function $u_{\varepsilon \delta}$ is said to be the solution of the problem (3.8)-(3.10) if for every $\varphi \in C^{\infty}\left(\overline{\Omega \backslash B_{\delta}}\right)$ and $\operatorname{dist}\left\{\operatorname{supp} \varphi, \partial B_{\delta}\right\}>0, u_{\varepsilon \delta}$ satisfies

$$
\int_{\Omega \backslash B_{\delta}}|x|^{\alpha}\left(\left|\nabla u_{\varepsilon \delta}\right|^{2}+\varepsilon\right)^{\frac{p-2}{2}} \nabla u_{\varepsilon \delta} \nabla \varphi d x-\int_{\partial \Omega} g \varphi d \sigma=\int_{\Omega \backslash B_{\delta}} f \varphi d x,
$$

and the condition (3.10) holds. 
Next we do the a priori estimates of $u_{\varepsilon \delta}$. For simplicity, we assume that $u_{\varepsilon \delta}$ is the classical solution of the problem (3.8)-(3.10); otherwise we only need to modify the coefficients of the equation (3.8), and then consider the modified equation.

Based on the extremum theorem, we have

$$
\max _{x \in \overline{\Omega \backslash B_{\delta}}}\left|u_{\varepsilon \delta}\right| \leq C
$$

where $C$ is independent of $\varepsilon$. Take $\varphi=u_{\varepsilon \delta}-\theta$ in (3.11). Then we have

$$
\int_{\Omega \backslash B_{\delta}}|x|^{\alpha}\left(\left|\nabla u_{\varepsilon \delta}\right|^{2}+\varepsilon\right)^{\frac{p-2}{2}}\left|\nabla u_{\varepsilon \delta}\right|^{2} d x \leq C,
$$

where $C$ is independent of $\varepsilon$. Furthermore, by the imbedding theorem, we also have

$$
\left\|u_{\varepsilon \delta}\right\|_{\beta_{0}} \leq C\left(\left\|\nabla u_{\varepsilon \delta}\right\|_{L^{p}}+\left\|\nabla u_{\varepsilon \delta}\right\|_{\infty}\right),
$$

since $p>n+\alpha$, where $\beta_{0}=1-\frac{n}{p}$.

Combining (3.12) with (3.13), we see that there exists a convergence subsequence of $\left\{u_{\varepsilon \delta}\right\}$ (for simplicity we still write it as $\left\{u_{\varepsilon \delta}\right\}$ ), a function $u_{\delta}$ and a vector $\vec{\zeta}=\left(\zeta_{1}, \ldots, \zeta_{n}\right)$, such that

$$
u_{\delta} \in C^{\beta}\left(\overline{\Omega \backslash B_{\delta}}\right) \quad \text { for } 0<\beta<\beta_{0}, \quad|x|^{-\alpha / p}|\vec{\zeta}| \in L^{p /(p-1)}\left(\Omega \backslash B_{\delta}\right)
$$

and

$$
\begin{gathered}
u_{\varepsilon \delta} \rightarrow u_{\delta}, \quad \text { uniformly in } \overline{\Omega \backslash B_{\delta}}, \\
\nabla u_{\varepsilon \delta} \rightarrow \nabla u_{\delta}, \quad \text { in } L^{p}\left(\Omega \backslash B_{\delta}\right), \\
|x|^{\alpha}\left(\left|\nabla u_{\varepsilon \delta}\right|^{2}+\varepsilon\right)^{\frac{p-2}{2}} \nabla u_{\varepsilon \delta} \rightarrow \vec{\zeta}, \quad \text { in } L^{p /(p-1)}\left(\Omega \backslash B_{\delta}\right) .
\end{gathered}
$$

The next step is to verify that $u_{\delta}$ is the solution of the problem (3.4)-(3.6). Let $\varepsilon \rightarrow 0^{+}$ in (3.11). Then

$$
\int_{\Omega \backslash B_{\delta}} \vec{\zeta} \nabla \varphi d x=\int_{\Omega \backslash B_{\delta}} f \varphi d x+\int_{\partial \Omega} g \varphi d \sigma .
$$

In order to prove that $u_{\delta}$ satisfies (3.7), we need only prove that for every $\varphi \in C^{\infty}\left(\overline{\Omega \backslash B_{\delta}}\right)$ and $\operatorname{dist}\left\{\operatorname{supp} \varphi, \partial B_{\delta}\right\}>0$, it follows that

$$
\int_{\Omega \backslash B_{\delta}}|x|^{\alpha}\left|\nabla u_{\delta}\right|^{p-2} \nabla u_{\delta} \nabla \varphi d x=\int_{\Omega \backslash B_{\delta}} \vec{\zeta} \nabla \varphi d x .
$$

Set $0 \leq \psi \in C^{\infty}\left(\overline{\Omega \backslash B_{\delta}}\right)$ and $\psi=1$ on $\operatorname{supp} \varphi$. Letting $\varphi=\psi\left(u_{\varepsilon \delta}-\theta\right)$ in (3.11), we have

$$
\begin{aligned}
& \int_{\Omega \backslash B_{\delta}}\left(u_{\varepsilon \delta}-\theta\right)|x|^{\alpha}\left(\left|\nabla u_{\varepsilon \delta}\right|^{2}+\varepsilon\right)^{\frac{p-2}{2}} \nabla u_{\varepsilon \delta} \nabla \psi d x \\
& +\int_{\Omega \backslash B_{\delta}}|x|^{\alpha}\left(\left|\nabla u_{\varepsilon \delta}\right|^{2}+\varepsilon\right)^{\frac{p-2}{2}}\left|\nabla u_{\varepsilon \delta}\right|^{2} \psi d x \\
& -\int_{\partial \Omega} g\left(\psi\left(u_{\varepsilon \delta}-\theta\right)\right) d \sigma=\int_{\Omega \backslash B_{\delta}} f\left(\psi\left(u_{\varepsilon \delta}-\theta\right)\right) d x .
\end{aligned}
$$

Set $v \in C^{\beta}\left(\overline{\Omega \backslash B_{\delta}}\right)$ and $|x|^{\alpha}|\nabla v|^{p} \in L^{1}\left(\Omega \backslash B_{\delta}\right)$. It is obvious that

$$
\int_{\Omega \backslash B_{\delta}} \psi|x|^{\alpha}\left(\left|\nabla u_{\varepsilon \delta}\right|^{2}+\varepsilon\right)^{(p-2) / 2}\left|\nabla u_{\varepsilon \delta}\right|^{2} d x
$$




$$
\begin{aligned}
& -\int_{\Omega \backslash B_{\delta}} \psi|x|^{\alpha}\left(|\nabla v|^{2}+\varepsilon\right)^{(p-2) / 2} \nabla v \nabla u_{\varepsilon \delta} d x \\
& -\int_{\Omega \backslash B_{\delta}} \psi|x|^{\alpha}\left(\left|\nabla u_{\varepsilon \delta}\right|^{2}+\varepsilon\right)^{(p-2) / 2} \nabla u_{\varepsilon \delta} \nabla v d x \\
& +\int_{\Omega \backslash B_{\delta}} \psi|x|^{\alpha}\left(|\nabla v|^{2}+\varepsilon\right)^{(p-2) / 2}|\nabla v|^{2} d x \geq 0 .
\end{aligned}
$$

This combined with 3.17) gives

$$
\begin{aligned}
& \int_{\partial \Omega} g\left(\psi\left(u_{\varepsilon \delta}-\theta\right)\right) d \sigma+\int_{\Omega \backslash B_{\delta}} f\left(\psi\left(u_{\varepsilon \delta}-\theta\right)\right) d x \\
& -\int_{\Omega \backslash B_{\delta}}\left(u_{\varepsilon \delta}-\theta\right)|x|^{\alpha}\left(\left|\nabla u_{\varepsilon \delta}\right|^{2}+\varepsilon\right)^{\frac{p-2}{2}} \nabla u_{\varepsilon \delta} \nabla \psi d x \\
& -\int_{\Omega \backslash B_{\delta}} \psi|x|^{\alpha}\left(\left|\nabla u_{\varepsilon \delta}\right|^{2}+\varepsilon\right)^{\frac{p-2}{2}} \nabla u_{\varepsilon \delta} \nabla v d x \\
& -\int_{\Omega \backslash B_{\delta}} \psi|x|^{\alpha}\left(|\nabla v|^{2}+\varepsilon\right)^{(p-2) / 2} \nabla v\left(\nabla u_{\varepsilon \delta}-\nabla v\right) d x \geq 0 .
\end{aligned}
$$

If

$$
\begin{aligned}
& \int_{\Omega \backslash B_{\delta}} \psi|x|^{\alpha}\left(|\nabla v|^{2}+\varepsilon\right)^{(p-2) / 2} \nabla v\left(\nabla u_{\varepsilon \delta}-\nabla v\right) d x \\
\rightarrow & \int_{\Omega \backslash B_{\delta}} \psi|x|^{\alpha}|\nabla v|^{p-2} \nabla v\left(\nabla u_{\delta}-\nabla v\right) d x
\end{aligned}
$$

as $\varepsilon \rightarrow 0$, then letting $\varepsilon \rightarrow 0$, we arrive at

$$
\begin{aligned}
& \int_{\partial \Omega} g \psi\left(u_{\delta}-\theta\right) d \sigma+\int_{\Omega \backslash B_{\delta}} f \psi\left(u_{\delta}-\theta\right) d x \\
& -\int_{\Omega \backslash B_{\delta}}\left(u_{\delta}-\theta\right) \vec{\zeta} \nabla \psi d x-\int_{\Omega \backslash B_{\delta}} \psi \vec{\zeta} \nabla v d x \\
& -\int_{\Omega \backslash B_{\delta}} \psi|x|^{\alpha}|\nabla v|^{p-2} \nabla v\left(\nabla u_{\delta}-\nabla v\right) d x \geq 0 .
\end{aligned}
$$

Indeed, if $p \geq 2$, the above limit relation (3.18) is obvious, while if $p<2$, note that

$$
\begin{aligned}
& \int_{\Omega \backslash B_{\delta}} \psi|x|^{\alpha}\left(\left(|\nabla v|^{2}+\varepsilon\right)^{(p-2) / 2}-|\nabla v|^{p-2}\right) \nabla v\left(\nabla u_{\varepsilon \delta}-\nabla v\right) d x \\
& \quad=\iint_{\left\{\Omega \backslash B_{\delta}:|\nabla v| \leq \sqrt{\varepsilon}\right\}} \psi|x|^{\alpha}\left(\left(|\nabla v|^{2}+\varepsilon\right)^{(p-2) / 2}-|\nabla v|^{p-2}\right) \nabla v\left(\nabla u_{\varepsilon \delta}-\nabla v\right) d x \\
& +\iint_{\left\{\Omega \backslash B_{\delta}:|\nabla v|>\sqrt{\varepsilon}\right\}} \psi|x|^{\alpha}\left(\left(|\nabla v|^{2}+\varepsilon\right)^{(p-2) / 2}-|\nabla v|^{p-2}\right) \nabla v\left(\nabla u_{\varepsilon \delta}-\nabla v\right) d x \\
& \quad \equiv I_{1}+I_{2} .
\end{aligned}
$$

Obviously, we have $I_{1} \rightarrow 0$ as $\varepsilon \rightarrow 0$. As for $I_{2}$, noticing that $|\nabla v|>\sqrt{\varepsilon}$, we have

$$
\begin{aligned}
\left|\left(\left(|\nabla v|^{2}+\varepsilon\right)^{(p-2) / 2}-|\nabla v|^{p-2}\right) \nabla v\right| & \leq \frac{2-p}{2} \varepsilon|\nabla v|^{p-3} \\
& \leq \frac{2-p}{2} \varepsilon^{(p-1) / 2} \rightarrow 0
\end{aligned}
$$


as $\varepsilon \rightarrow 0$. Summing up, (3.18) holds for any $p>n+\alpha$.

Choosing $\varphi=\psi\left(u_{\delta}-\theta\right)$ in (3.15), we have

$$
\begin{aligned}
& \int_{\Omega \backslash B_{\delta}} f \psi\left(u_{\delta}-\theta\right) d x+\int_{\partial \Omega} g \psi\left(u_{\delta}-\theta\right) d \sigma \\
& -\int_{\Omega \backslash B_{\delta}}\left(u_{\delta}-\theta\right) \vec{\zeta} \nabla \psi d x=\int_{\Omega \backslash B_{\delta}} \psi \vec{\zeta} \nabla\left(u_{\delta}-\theta\right) d x .
\end{aligned}
$$

Substituting the above equation into (3.19), we deduce that

$$
\begin{aligned}
& \int_{\Omega \backslash B_{\delta}} \psi \vec{\zeta} \nabla\left(u_{\delta}-\theta\right) d x-\int_{\Omega \backslash B_{\delta}} \psi \vec{\zeta} \nabla v d x \\
& -\int_{\Omega \backslash B_{\delta}} \psi|x|^{\alpha}|\nabla v|^{p-2} \nabla v\left(\nabla u_{\delta}-\nabla v\right) d x \geq 0,
\end{aligned}
$$

that is,

$$
\int_{\Omega \backslash B_{\delta}} \psi\left(\vec{\zeta}-|x|^{\alpha}|\nabla v|^{p-2} \nabla v\right)\left(\nabla u_{\delta}-\nabla v\right) d x \geq 0
$$

Choosing $v=u_{\delta}-\lambda \varphi$ and $\lambda>0$ in the above inequality, we have

SO

$$
\int_{\Omega \backslash B_{\delta}} \psi\left(\vec{\zeta}-|x|^{\alpha}\left|\nabla\left(u_{\delta}-\lambda \varphi\right)\right|^{p-2} \nabla\left(u_{\delta}-\lambda \varphi\right)\right)\left(\nabla u_{\delta}-\nabla\left(u_{\delta}-\lambda \varphi\right)\right) d x \geq 0
$$

$$
\int_{\Omega \backslash B_{\delta}} \psi\left(\vec{\zeta}-|x|^{\alpha}\left|\nabla\left(u_{\delta}-\lambda \varphi\right)\right|^{p-2} \nabla\left(u_{\delta}-\lambda \varphi\right)\right) \nabla \varphi d x \geq 0 .
$$

Letting $\lambda \rightarrow 0^{+}$, then we have

$$
\int_{\Omega \backslash B_{\delta}} \psi\left(\vec{\zeta}-|x|^{\alpha}\left|\nabla u_{\delta}\right|^{p-2} \nabla u_{\delta}\right) \nabla \varphi d x \geq 0 .
$$

Using the similar methods above, we obtain the inverse inequality for $\lambda<0$. Hence

$$
\int_{\Omega \backslash B_{\delta}} \psi\left(\vec{\zeta}-|x|^{\alpha}\left|\nabla u_{\delta}\right|^{p-2} \nabla u_{\delta}\right) \nabla \varphi d x=0 .
$$

Noticing that $\psi=1$ on $\operatorname{supp} \varphi$, then (3.16) is satisfied.

Falling back to the condition (3.10) and using that $u_{\varepsilon \delta}$ tends to $u_{\delta}$ uniformly on $\overline{\Omega \backslash B_{\delta}}$, we see that $u_{\delta}(x)=\theta$, which implies the condition (3.6).

Finally, we prove the uniqueness of the solutions. Let $u$ and $v$ be the two solutions. Then

$$
\int_{\Omega \backslash B_{\delta}}|x|^{\alpha}\left(|\nabla u|^{p-2} \nabla u-|\nabla v|^{p-2} \nabla v\right) \nabla \varphi d x=0 .
$$

Choosing $\varphi=u-v$ in the above equation, it follows that

$$
\int_{\Omega \backslash B_{\delta}}|x|^{\alpha}\left(|\nabla u|^{p-2} \nabla u-|\nabla v|^{p-2} \nabla v\right) \nabla(u-v) d x=0 .
$$

Since

$$
\left(|\nabla u|^{p-2} \nabla u-|\nabla v|^{p-2} \nabla v\right)(\nabla u-\nabla v) \geq 0
$$

we have

$$
\nabla u=\nabla v, \quad \text { a.e. in } \Omega \backslash B_{\delta} .
$$


Thanks to the condition (3.10), we obtain

$$
u(x)=v(x), \quad x \in \Omega \backslash B_{\delta} .
$$

The proof is complete.

In order to prove Theorem 3.2, we firstly need to establish the comparison theorem. Considering the process of constructing the approximate solutions, applying the comparison theorem of the classical solutions to the approximate problem (see Chapter 4 in [18), we obtain

Lemma 3.4. Let $u, v \in C\left(\overline{\Omega \backslash B_{\delta}}\right) \cap W^{1, p}\left(\Omega \backslash B_{\delta}\right)$, for any $0<\delta<1$. If $\mathcal{L} u \leq \mathcal{L} v$ on $\Omega \backslash B_{\delta}$ and $\left.u\right|_{\partial\left(\Omega \backslash B_{\delta}\right)} \leq\left. v\right|_{\partial\left(\Omega \backslash B_{\delta}\right)}$, then

$$
u(x) \leq v(x), \quad \forall x \in \Omega \backslash B_{\delta},
$$

where $\mathcal{L} u \equiv-\operatorname{div}\left(|x|^{\alpha}|\nabla u|^{p-2} \nabla u\right)=f(x)$.

Now we prove the main results.

Proof of Theorem 3.2. For any $\varphi \in C^{\infty}(\bar{\Omega})$, since $\operatorname{dist}\{\operatorname{supp} \varphi, 0\}>0$, we choose $\delta$ such that $\delta=\frac{1}{2} \operatorname{dist}\{\operatorname{supp} \varphi, 0\}>0$ in $B_{\delta}$. By using the boundedness of the $L^{p}$ norm of $|x|^{\alpha / p} \nabla u_{\delta}$, we get the boundedness of the $L^{p}$ norm of $|x|^{\alpha / p} \nabla u$. Thus we have

$$
\int_{\Omega \backslash B_{\delta}}|x|^{\alpha}\left|\nabla u_{\delta}\right|^{p-2} \nabla u_{\delta} \nabla \varphi d x \rightarrow \int_{\Omega}|x|^{\alpha}|\nabla u|^{p-2} \nabla u \nabla \varphi d x
$$

as $\delta \rightarrow 0$. Therefore, by applying the equation (3.7), it is easy to see that $u$ satisfies the equation (3.1) and the boundary value condition (3.2) in the sense of distribution.

Next we verify the condition (3.3). Assume $B_{1 / 2} \subset \Omega$. We consider the problem on the domain $B_{1 / 2} \backslash B_{\delta}$.

$$
\begin{gathered}
-\operatorname{div}\left(|x|^{\alpha}|\nabla u|^{p-2} \nabla u\right)=f(x), \quad x \in B_{1 / 2} \backslash B_{\delta}, \\
u(x)=\theta, \quad x \in \partial B_{\delta}, \\
u(x)=h(x), \quad x \in \partial B_{1 / 2} .
\end{gathered}
$$

Utilizing the results on the domain $\Omega \backslash B_{\delta}$, it follows that

$$
N \leq u(x) \leq M, \quad x \in \Omega \backslash B_{\delta},
$$

where $N$ and $M$ are both constants which depend on the bounds of $f$ and $g$. Therefore $N \leq u(x) \leq M$ on $\partial B_{1 / 2}$. Now we consider the radially symmetric solutions of the following two problems:

$$
\begin{gathered}
-\operatorname{div}\left(|x|^{\alpha}|\nabla w|^{p-2} \nabla w\right)=h_{1}(|x|), \quad x \in B_{1 / 2} \backslash B_{\delta}, \\
w(x)=\theta, \quad x \in \partial B_{\delta}, \\
w(x)=N, \quad x \in \partial B_{1 / 2}
\end{gathered}
$$

and

$$
\begin{gathered}
-\operatorname{div}\left(|x|^{\alpha}|\nabla v|^{p-2} \nabla v\right)=h_{2}(|x|), \quad x \in B_{1 / 2} \backslash B_{\delta}, \\
v(x)=\theta, \quad x \in \partial B_{\delta}, \\
v(x)=M, \quad x \in \partial B_{1 / 2} .
\end{gathered}
$$


It is obvious that $v$ and $w$ are the sup-solution and the sub-solution, respectively, of the problem (3.20)-(3.22). Let $r=|x|$. Problems (3.23)-(3.25) and (3.26)-(3.28) can be rewritten as

$$
\begin{gathered}
\left(r^{n-1+\alpha} \phi_{p}\left(w^{\prime}\right)\right)^{\prime}=-r^{n-1} h_{1}(r), \quad r \in(\delta, 1 / 2), \\
w(1 / 2)=N, \\
w(\delta)=\theta
\end{gathered}
$$

and

$$
\begin{gathered}
\left(r^{n-1+\alpha} \phi_{p}\left(v^{\prime}\right)\right)^{\prime}=-r^{n-1} h_{2}(r), \quad r \in(\delta, 1 / 2), \\
v(1 / 2)=M, \\
v(\delta)=\theta .
\end{gathered}
$$

By analyzing the two radially symmetric problems above, we know that there exist $\sigma_{1}$ and $\sigma_{2}$, such that $w^{\prime}\left(\sigma_{1}\right)=(1 / 2-\delta)(N-\theta), v^{\prime}\left(\sigma_{2}\right)=(1 / 2-\delta)(M-\theta)$,

$$
w(r)=\left\{\begin{array}{r}
\theta+\int_{\delta}^{r} \frac{1}{s^{\frac{n-1+\alpha}{p-1}}} \phi_{q}\left(\sigma_{1}^{n-1+\alpha} \phi_{p}((1 / 2-\delta)(N-\theta))+\int_{s}^{\sigma_{1}} t^{n-1} h_{1}(t) d t\right) d s, \\
\text { if } \delta \leq r \leq \sigma_{1}, \\
N+\int_{r}^{1} \frac{1}{s^{\frac{n-1+\alpha}{p-1}}} \phi_{q}\left(\sigma_{1}^{n-1+\alpha} \phi_{p}((1 / 2-\delta)(\theta-N))+\int_{\sigma_{1}}^{s} t^{n-1} h_{1}(t) d t\right) d s, \\
\quad \text { if } \sigma_{1}<r \leq 1,
\end{array}\right.
$$

and

$$
v(r)=\left\{\begin{array}{r}
\theta+\int_{\delta}^{r} \frac{1}{s^{\frac{n-1+\alpha}{p-1}}} \phi_{q}\left(\sigma_{2}^{n-1+\alpha} \phi_{p}((1 / 2-\delta)(M-\theta))+\int_{s}^{\sigma_{2}} t^{n-1} h_{2}(t) d t\right) d s, \\
\text { if } \delta \leq r \leq \sigma_{2}, \\
M+\int_{r}^{1} \frac{1}{s^{\frac{n-1+\alpha}{p-1}}} \phi_{q}\left(\sigma_{2}^{n-1+\alpha} \phi_{p}((1 / 2-\delta)(\theta-M))+\int_{\sigma_{2}}^{s} t^{n-1} h_{2}(t) d t\right) d s, \\
\text { if } \sigma_{2}<r \leq 1 .
\end{array}\right.
$$

Since $p>n+\alpha>1, \alpha>0$, it follows that $0<\frac{n-1+\alpha}{p-1}<1$. Hence, when $\delta \rightarrow 0$, both $w(r)$ and $v(r)$ exist on $[0,1]$, and

$$
\lim _{x \rightarrow 0} w(x)=\theta=\lim _{x \rightarrow 0} v(x) .
$$

Therefore,

$$
\lim _{x \rightarrow 0} u(x)=\theta .
$$

Finally, we consider the uniqueness of the solutions for the problem (3.1)-(3.3). Let $u$ and $v$ be the two solutions of (3.1)-(3.3). Then

$$
\int_{\Omega}|x|^{\alpha}\left(|\nabla u|^{p-2} \nabla u-|\nabla v|^{p-2} \nabla v\right) \nabla \varphi d x=0
$$


When $p>n+\alpha$ and $\alpha>0$, using the embedding theorem in the weighted Sobolev space (see Theorem 9.15 in [19]), we know that the test function in the above equation can be chosen as $\varphi=u-v$; hence

$$
\int_{\Omega}|x|^{\alpha}\left(|\nabla u|^{p-2} \nabla u-|\nabla v|^{p-2} \nabla v\right) \nabla(u-v) d x=0 .
$$

Since

$$
\left(|\nabla u|^{p-2} \nabla u-|\nabla v|^{p-2} \nabla v\right)(\nabla u-\nabla v) \geq 0
$$

we have

$$
\nabla u=\nabla v, \quad \text { a.e. in } \Omega .
$$

Thanks to the condition (3.3), it is easy to see that

$$
u(x)=v(x), \quad x \in \Omega .
$$

3.2. Unremovable orifice. For the problem (3.1)-(3.3), under the condition $M_{1}+$ $\int_{0}^{1} r^{n-1} h_{1}(r) d r>0$ or $M_{2}+\int_{0}^{1} r^{n-1} h_{2}(r) d r<0$, we gain the properties of the orifice.

ThEOREM 3.5. If $p>n+\alpha$, under one of the conditions

or

$$
M_{1}+\int_{0}^{1} r^{n-1} h_{1}(r) d r>0
$$

$$
M_{2}+\int_{0}^{1} r^{n-1} h_{2}(r) d r<0,
$$

the origin is an unremovable orifice.

Proof. Falling back to the proof of Theorem 2.6, we see that if $M_{1}+\int_{0}^{1} r^{n-1} h_{1}(r) d r>0$ or $M_{2}+\int_{0}^{1} r^{n-1} h_{2}(r) d r<0, u$ cannot satisfy the equation (3.1) and the boundary value condition (3.2) in the sense of distribution.

4. Evolutionary solution. In this section, we are now in a position to investigate the evolutionary problem, namely the problem (1.1)-(1.3) with the following additional condition on $x=0$ :

$$
\lim _{x \rightarrow 0} u(x, t)=\theta, \quad t \in(0, T)
$$

where $\theta$ is a constant.

Assume that $N_{1} \leq f(x, t) \leq N_{2}$ and $M_{1} \leq g(x, t) \leq M_{2}$, where $N_{i}$ and $M_{i}$ are all constants, $i=1,2$.

Definition 4.1. A function $u$ is said to be a solution of the problem (1.1), (1.2), (1.3) and (4.1), if $u \in C\left(\overline{Q_{T}}\right)$, for every $0<\delta<1, u \in W^{1, p}\left(R_{\delta T}\right), \frac{\partial u}{\partial t} \in L^{2}\left(Q_{T}\right)$, $|x|^{\alpha}\left|\nabla^{*} u\right|^{p} \in L^{1}\left(Q_{T}\right)$, and $u$ satisfies

$$
\iint_{Q_{T}}\left(\frac{\partial u}{\partial t} \varphi+|x|^{\alpha}\left|\nabla^{*} u\right|^{p-2} \nabla^{*} u \nabla \varphi\right) d x d t-\int_{0}^{T} \int_{\partial \Omega} g \varphi d \sigma d t=\iint_{Q_{T}} f \varphi d x d t,
$$

for any $\varphi \in C^{\infty}\left(\overline{Q_{T}}\right)$ with dist $\{\operatorname{supp} \varphi, 0\}>0$, where $Q_{T}=\Omega \times(0, T), R_{\delta T}=\left(\Omega \backslash B_{\delta}\right) \times$ $(0, T), \nabla^{*} u$ is the generalized gradient of $u$ in $(\Omega \backslash\{0\}) \times(0, T)$, and the conditions (1.2) and (4.1) hold. 


\subsection{Existence and uniqueness.}

Theorem 4.2. When $p>n+\alpha$, the problem (1.1), (1.2), (1.3) and (4.1) admits a unique solution.

Firstly, we consider the problem on the domain $X_{T}=F \times(0, T)$, where $F=\Omega \backslash B_{\delta}$, $\delta>0$ is a constant which is small enough, and

$$
\begin{gathered}
\frac{\partial u_{\delta}}{\partial t}-\operatorname{div}\left(|x|^{\alpha}\left|\nabla u_{\delta}\right|^{p-2} \nabla u_{\delta}\right)=f(x, t), \quad(x, t) \in X_{T}, \\
|x|^{\alpha}\left|\nabla u_{\delta}\right|^{p-2} \nabla u_{\delta} \cdot \vec{\nu}=g(x, t), \quad(x, t) \in \partial \Omega \times(0, T), \\
u_{\delta}(x, t)=\theta, \quad(x, t) \in \partial B_{\delta} \times(0, T), \\
u_{\delta}(x, 0)=u_{\delta 0}(x), \quad x \in F .
\end{gathered}
$$

The function $u_{\delta}$ is said to be a solution of the problem (4.2)-(4.5) if $u_{\delta} \in C^{\beta}\left(\overline{X_{T}}\right) \cap$ $W^{1, p}\left(X_{T}\right), \frac{\partial u}{\partial t} \in L^{2}\left(X_{T}\right),|x|^{\alpha}\left|\nabla u_{\delta}\right|^{p} \in L^{1}\left(X_{T}\right)$, and $u$ satisfies

$$
\begin{aligned}
& \iint_{X_{T}}\left(\frac{\partial u_{\delta}}{\partial t} \varphi+|x|^{\alpha}\left|\nabla u_{\delta}\right|^{p-2} \nabla u_{\delta} \nabla \varphi\right) d x d t \\
& -\int_{0}^{T} \int_{\partial \Omega} g \varphi d \sigma d t=\iint_{X_{T}} f \varphi d x d t
\end{aligned}
$$

for every test function $\varphi \in C^{\infty}\left(\overline{X_{T}}\right)$ with $\operatorname{dist}\left\{\operatorname{supp} \varphi, \partial B_{\delta}\right\}>0,0<\beta<1$, and the conditions (4.5) and (4.4) are satisfied in the usual sense.

In order to study the existence and the uniqueness of solutions for the problem (1.1), (1.2), (1.3) and (4.1), we need the following lemma.

Lemma 4.3. Assume that $p>n+\alpha$, and $u_{0}, f, g$ are smooth enough.

i) When $p \geq 2,|x|^{\alpha}\left|\nabla u_{0}\right|^{p} \in L^{1}(F)$.

ii) When $n+\alpha<p<2, u_{0}$ is smooth enough and with compact support.

Then the problem (4.2)- (4.5) admits a unique solution.

Proof. For every $\varepsilon>0$, considering the approximating problem

$$
\begin{gathered}
\frac{\partial u_{\varepsilon \delta}}{\partial t}-\operatorname{div}\left(|x|^{\alpha}\left(\left|\nabla u_{\varepsilon \delta}\right|^{2}+\varepsilon\right)^{\frac{p-2}{2}} \nabla u_{\varepsilon \delta}\right)=f(x, t), \quad(x, t) \in X_{T}, \\
|x|^{\alpha}\left(\left|\nabla u_{\varepsilon \delta}\right|^{2}+\varepsilon\right)^{\frac{p-2}{2}} \nabla u_{\varepsilon \delta} \cdot \vec{\nu}=g(x, t), \quad(x, t) \in \partial \Omega \times(0, T), \\
u_{\varepsilon \delta}(x, t)=\theta, \quad(x, t) \in \partial B_{\delta} \times(0, T), \\
u_{\varepsilon \delta}(x, 0)=u_{\varepsilon \delta 0}(x), \quad x \in F .
\end{gathered}
$$

Here $u_{\varepsilon \delta 0}(x)$ is smooth enough and approximates $u_{\delta 0}(x)$ uniformly as $\varepsilon \rightarrow 0$.

According to the classical parabolic theory (see for example [20]), there exists a unique solution $u_{\varepsilon \delta} \in C^{\beta}\left(\overline{X_{T}}\right) \cap L^{p}\left(0, T ; W^{1, p}(F)\right)$ of the problem (4.7) (4.10), and the solution satisfies $\frac{\partial u_{\varepsilon \delta}}{\partial t} \in L^{2}\left(X_{T}\right)$. Here, we call $u_{\varepsilon \delta}$ the solution of the problem (4.7)-(4.10) if for 
every $\varphi \in C^{\infty}\left(\overline{X_{T}}\right)$ with $\operatorname{dist}\left\{\operatorname{supp} \varphi, \partial B_{\delta}\right\}>0$, it follows that

$$
\begin{aligned}
& \iint_{X_{T}}\left(\frac{\partial u_{\varepsilon \delta}}{\partial t} \varphi+|x|^{\alpha}\left(\left|\nabla u_{\varepsilon \delta}\right|^{2}+\varepsilon\right)^{\frac{p-2}{2}} \nabla u_{\varepsilon \delta} \nabla \varphi\right) d x d t \\
& -\int_{0}^{T} \int_{\partial \Omega} g \varphi d \sigma d t=\iint_{X_{T}} f \varphi d x d t
\end{aligned}
$$

and conditions (4.9) and (4.10) hold.

Next we do the a priori estimates of $u_{\varepsilon \delta}$. For simplicity, we assume that $u_{\varepsilon \delta}$ is the classical solution of the problem (4.7)-(4.10). Otherwise, we need only to modify the coefficient of the equation (4.7) and then consider the modified equation.

By the extremum theorem, we have

$$
\max _{(x, t) \in \overline{X_{T}}}\left|u_{\varepsilon \delta}\right| \leq C
$$

where $C$ is independent of $\varepsilon$.

By an approximating process, we take $\varphi=u_{\varepsilon \delta}-\theta$ in (4.11). Then

$$
\begin{aligned}
& \iint_{X_{T}} \frac{\partial\left(u_{\varepsilon \delta}-\theta\right)}{\partial t}\left(u_{\varepsilon \delta}-\theta\right) d x d t \\
& +\iint_{X_{T}}|x|^{\alpha}\left(\left|\nabla u_{\varepsilon \delta}\right|^{2}+\varepsilon\right)^{\frac{p-2}{2}}\left|\nabla u_{\varepsilon \delta}\right|^{2} d x d t \\
= & \iint_{X_{T}} f\left(u_{\varepsilon \delta}-\theta\right) d x d t+\int_{0}^{T} \int_{\partial \Omega} g\left(u_{\varepsilon \delta}-\theta\right) d \sigma d t .
\end{aligned}
$$

Therefore,

$$
\begin{aligned}
& \iint_{X_{T}}|x|^{\alpha}\left(\left|\nabla u_{\varepsilon \delta}\right|^{2}+\varepsilon\right)^{\frac{p-2}{2}}\left|\nabla u_{\varepsilon \delta}\right|^{2} d x d t \\
= & \iint_{X_{T}} f\left(u_{\varepsilon \delta}-\theta\right) d x d t+\int_{0}^{T} \int_{\partial \Omega} g\left(u_{\varepsilon \delta}-\theta\right) d \sigma d t \\
& -\iint_{X_{T}} \frac{\partial\left(u_{\varepsilon \delta}-\theta\right)}{\partial t}\left(u_{\varepsilon \delta}-\theta\right) d x d t \\
= & \iint_{X_{T}} f\left(u_{\varepsilon \delta}-\theta\right) d x d t+\int_{0}^{T} \int_{\partial \Omega} g\left(u_{\varepsilon \delta}-\theta\right) d \sigma d t \\
& -\frac{1}{2} \iint_{X_{T}} \frac{\partial\left(u_{\varepsilon \delta}-\theta\right)^{2}}{\partial t} d x d t \\
\leq & \iint_{X_{T}}\left|f\left(u_{\varepsilon \delta}-\theta\right)\right| d x d t+\int_{0}^{T} \int_{\partial \Omega}\left|g\left(u_{\varepsilon \delta}-\theta\right)\right| d \sigma d t \\
& -\frac{1}{2} \int_{F}\left(u_{\varepsilon \delta}-\theta\right)^{2}(x, T) d x+\frac{1}{2} \int_{F}\left(u_{\varepsilon \delta}-\theta\right)^{2}(x, 0) d x \leq C,
\end{aligned}
$$

where $C$ is independent of $\varepsilon$. 
We divide into two cases $(p \geq 2$ and $n+\alpha<p<2)$ to do the a priori estimates of $\frac{\partial u_{\varepsilon \delta}}{\partial t}$ in $L^{2}\left(0, T ; L_{\text {loc }}^{2}(F)\right)$, i.e.

$$
\iint_{X_{T}} \xi\left(\frac{\partial u_{\varepsilon \delta}}{\partial t}\right)^{2} d x d t \leq C
$$

and in $L^{\infty}\left(0, T ; L^{1}(F)\right)$, i.e.,

$$
\sup _{0<t<T} \int_{F}\left|\frac{\partial u_{\varepsilon \delta}}{\partial t}\right| d x \leq C
$$

where $C$ is independent of $\varepsilon, \xi \in C_{0}^{\infty}(F)$ such that $\xi=1$ on $F^{\prime}, 0 \leq \xi \leq 1$ and $|\nabla \xi| \leq$ $\tilde{C} / \eta$. Here $\eta$ is a positive constant which is small enough, $F^{\prime}=\{x \neq 0:$ dist $(x, \partial F)>\eta\}$, and $\tilde{C}$ is a constant which is independent of $\varepsilon, \delta$ and $\eta$.

Case 1. $p \geq 2$. We take $\varphi=\xi \frac{\partial\left(u_{\varepsilon \delta}-\theta\right)}{\partial t}$ in (4.11), use Hölder's inequality, and notice that $p \geq 2$; then (4.14) is obtained. To verify 4.15), let

$$
\begin{gathered}
H_{\eta}(s)=\int_{0}^{s} h_{\eta}(t) d t, \\
h_{\eta}(s)= \begin{cases}\frac{2}{\eta}\left(1-\frac{|s|}{\eta}\right), & |s|<\eta, \\
0, & |s| \geq \eta .\end{cases}
\end{gathered}
$$

It is easy to see that

$$
\begin{aligned}
& h_{\eta}(s) \geq 0, \quad\left|s h_{\eta}(s)\right| \leq 1, \quad\left|H_{\eta}(s)\right| \leq 1, \\
& \lim _{\eta \rightarrow 0^{+}} H_{\eta}(s)=\operatorname{sgn}(s), \quad \lim _{\eta \rightarrow 0^{+}} s h_{\eta}(s)=0 .
\end{aligned}
$$

Differentiating (4.7) with respect to $t$, it follows that

$$
\begin{aligned}
& \frac{\partial v_{\varepsilon}}{\partial t}-\operatorname{div}\left(|x|^{\alpha}\left(\left|\nabla u_{\varepsilon \delta}\right|^{2}+\varepsilon\right)^{\frac{p-2}{2}} \nabla v_{\varepsilon}\right) \\
& -(p-2) \operatorname{div}\left(|x|^{\alpha}\left(\left|\nabla u_{\varepsilon \delta}\right|^{2}+\varepsilon\right)^{\frac{p-4}{2}}\left(\nabla u_{\varepsilon \delta} \nabla v_{\varepsilon}\right) \nabla u_{\varepsilon \delta}\right)=\frac{\partial f}{\partial t},
\end{aligned}
$$

where $v_{\varepsilon}=\frac{\partial u_{\varepsilon \delta}}{\partial t}$. Multiplying $H_{\eta}\left(v_{\varepsilon}\right)$ by the above equation, integrating with respect to $x$ over $F$, and then integrating by parts, we have

$$
\begin{aligned}
& \int_{F} \frac{\partial v_{\varepsilon}}{\partial t} H_{\eta}\left(v_{\varepsilon}\right) d x+\int_{F}|x|^{\alpha}\left(\left|\nabla u_{\varepsilon \delta}\right|^{2}+\varepsilon\right)^{\frac{p-2}{2}} h_{\eta}\left(v_{\varepsilon}\right) \nabla v_{\varepsilon} \nabla v_{\varepsilon} d x \\
& +(p-2) \int_{F}|x|^{\alpha}\left(\left|\nabla u_{\varepsilon \delta}\right|^{2}+\varepsilon\right)^{\frac{p-4}{2}}\left(\nabla u_{\varepsilon \delta} \nabla v_{\varepsilon}\right) h_{\eta}\left(v_{\varepsilon}\right) \nabla u_{\varepsilon \delta} \nabla v_{\varepsilon} d x \\
& =\int_{F} \frac{\partial f}{\partial t} H_{\eta}\left(v_{\varepsilon}\right) d x .
\end{aligned}
$$

Hence

$$
\begin{aligned}
& \frac{\partial}{\partial t} \int_{F} \Theta_{\eta}\left(v_{\varepsilon}\right) d x \\
= & \int_{F} \frac{\partial f}{\partial t} H_{\eta}\left(v_{\varepsilon}\right) d x-\int_{F}|x|^{\alpha}\left(\left|\nabla u_{\varepsilon \delta}\right|^{2}+\varepsilon\right)^{\frac{p-2}{2}} h_{\eta}\left(v_{\varepsilon}\right)\left|\nabla v_{\varepsilon}\right|^{2} d x
\end{aligned}
$$




$$
-(p-2) \int_{F}|x|^{\alpha}\left(\left|\nabla u_{\varepsilon \delta}\right|^{2}+\varepsilon\right)^{\frac{p-4}{2}} h_{\eta}\left(v_{\varepsilon}\right)\left(\nabla u_{\varepsilon \delta} \nabla v_{\varepsilon}\right)^{2} d x \leq\left\|\frac{\partial f}{\partial t}\right\|_{L^{1}(F)},
$$

where

$$
\Theta_{\eta}(s)=\int_{0}^{s} H_{\eta}(\sigma) d \sigma
$$

Letting $\eta \rightarrow 0^{+}$, it follows that

$$
\frac{\partial}{\partial t} \int_{F}\left|v_{\varepsilon}\right| d x \leq\left\|\frac{\partial f}{\partial t}\right\|_{L^{1}(F)} .
$$

Using Gronwall's inequality, (4.15) is obtained.

Case 2. $1<p<2$. In fact, we obtain the estimates of $\frac{\partial u_{\varepsilon \delta}}{\partial t}$ in $L^{\infty}\left(X_{T}\right)$. Since $u_{0}$ is smooth enough and with compact support, we assume that $\| \operatorname{div}\left(|x|^{\alpha}\left(\left|\nabla u_{\varepsilon \delta, 0}\right|^{2}\right.\right.$ $\left.+\varepsilon)^{(p-2) / 2} \nabla u_{\varepsilon \delta, 0}\right) \|_{L^{\infty}(F)}$ and $\left\|\nabla u_{\varepsilon \delta}\right\|_{L^{\infty}(F)}$ are bounded uniformly. Differentiating with respect to $t$ on both sides of (4.7) gives

$$
\frac{\partial v_{\varepsilon}}{\partial t}-a_{i j} D_{i j} v_{\varepsilon}-D_{j} a_{i j} D_{i} v_{\varepsilon}=\frac{\partial f}{\partial t},
$$

where $v_{\varepsilon}=\frac{\partial u_{\varepsilon \delta}}{\partial t}$

$$
\begin{aligned}
a_{i j}=|x|^{\alpha}\left(\left|\nabla u_{\varepsilon \delta}\right|^{2}+\varepsilon\right)^{\frac{p-2}{2}} \cdot\left(\delta_{i j}+(p-2)\left(\left|\nabla u_{\varepsilon \delta}\right|^{2}+\varepsilon\right)^{-1} D_{i} u_{\varepsilon \delta} D_{j} u_{\varepsilon \delta}\right), \\
\delta_{i j}= \begin{cases}1, & \text { if } i=j, \\
0, & \text { if } i \neq j .\end{cases}
\end{aligned}
$$

It is obvious that $v_{\varepsilon}(x, t)$ is bounded on $\partial F \times(0, T)$, and

$$
v_{\varepsilon}(x, 0)=\operatorname{div}\left(|x|^{\alpha}\left(\left|\nabla u_{\varepsilon, 0}\right|^{2}+\varepsilon\right)^{(p-2) / 2} \nabla u_{\varepsilon, 0}\right)+f, \quad x \in F .
$$

Let $B=|x|^{\alpha}\left(\left|\nabla u_{\varepsilon \delta}\right|^{2}+\varepsilon\right)^{(p-2) / 2}$. It is easy to see that for every $\xi=\left(\xi_{1}, \xi_{2}, \ldots, \xi_{n}\right) \in \mathbb{R}^{n}$, we have

$$
\min \{p-1,1\} B|\xi|^{2} \leq a_{i j} \xi_{i} \xi_{j} \leq \max \{p-1,1\} B|\xi|^{2} .
$$

By the extremum theorem, we have

$$
\left\|\frac{\partial u_{\varepsilon \delta}}{\partial t}\right\|_{L^{\infty}\left(X_{T}\right)} \leq C
$$

where $C$ is independent of $\varepsilon$, and the estimate implies that (4.14) and (4.15) are both established.

By (4.12) (4.15), there exist a subsequence of $\left\{u_{\varepsilon \delta}\right\}$ (for simplicity, we denote it by $\left\{u_{\varepsilon \delta}\right\}$ itself), a function $u_{\delta}$ and a vector $\vec{\zeta}=\left(\zeta_{1}, \ldots, \zeta_{n}\right)$, such that

$$
\begin{gathered}
u_{\delta} \in L^{\infty}\left(\overline{X_{T}}\right), \\
\frac{\partial u_{\delta}}{\partial t} \in L^{2}\left(0, T ; L_{\mathrm{loc}}^{2}(F)\right) \cap L^{1}\left(X_{T}\right), \quad|x|^{-\alpha / p}|\vec{\zeta}| \in L^{p /(p-1)}\left(X_{T}\right),
\end{gathered}
$$

and

$$
\begin{gathered}
u_{\varepsilon \delta} \rightarrow u \text { in } L^{r}\left(X_{T}\right) \text { for any } 1<r<+\infty, \\
\nabla u_{\varepsilon \delta} \rightarrow \nabla u \text { in } L^{p}\left(X_{T} ; \mathbb{R}^{n}\right),
\end{gathered}
$$




$$
\begin{gathered}
\frac{\partial u_{\varepsilon \delta}}{\partial t} \rightarrow \frac{\partial u}{\partial t} \text { in } L^{2}\left(X_{T}\right), \\
|x|^{\alpha}\left(\left|\nabla u_{\varepsilon \delta}\right|^{2}+\varepsilon\right)^{\frac{p-2}{2}} \nabla u_{\varepsilon \delta} \rightarrow \vec{\zeta} \text { in } L^{p /(p-1)}\left(X_{T} ; \mathbb{R}^{n}\right) .
\end{gathered}
$$

Next, we prove that $u_{\delta}$ is a solution of the equation (4.2) with the boundary value condition (4.3). Let $\varepsilon \rightarrow 0^{+}$in (4.11). For every $\varphi \in C^{\infty}\left(\overline{X_{T}}\right)$ with $\operatorname{dist}\left\{\operatorname{supp} \varphi, \partial B_{\delta}\right\}>$ 0 ,

$$
\iint_{X_{T}}\left(\frac{\partial u_{\delta}}{\partial t} \varphi+\vec{\zeta} \nabla \varphi\right) d x d t-\int_{0}^{T} \int_{\partial \Omega} g \varphi d \sigma d t=\iint_{X_{T}} f \varphi d x d t
$$

holds. Therefore, in order to prove that $u_{\delta}$ is the solution of (4.2) and (4.3), we only need to verify that

$$
\vec{\zeta}=|x|^{\alpha}\left|\nabla u_{\delta}\right|^{p-2} \nabla u_{\delta}
$$

In other words, we want to show that for every $\varphi \in C^{\infty}\left(\overline{X_{T}}\right)$ with $\operatorname{dist}\left\{\operatorname{supp} \varphi, \partial B_{\delta}\right\}>0$,

$$
\iint_{X_{T}}|x|^{\alpha}\left|\nabla u_{\delta}\right|^{p-2} \nabla u_{\delta} \nabla \varphi d x d t=\iint_{X_{T}} \vec{\zeta} \nabla \varphi d x d t
$$

Let $0 \leq \psi \in C^{\infty}\left(\overline{X_{T}}\right)$, dist $\left\{\operatorname{supp} \psi, \partial B_{\delta}\right\}>0$ and $\psi=1$ on $\operatorname{supp} \varphi$. Taking $\varphi=\psi\left(u_{\varepsilon \delta}-\theta\right)$ in (4.11), then

$$
\begin{aligned}
& \iint_{X_{T}} \frac{\partial\left(u_{\varepsilon \delta}-\theta\right)}{\partial t}\left(\psi\left(u_{\varepsilon \delta}-\theta\right)\right) d x d t \\
& +\iint_{X_{T}}|x|^{\alpha}\left(\left|\nabla u_{\varepsilon \delta}\right|^{2}+\varepsilon\right)^{\frac{p-2}{2}}\left|\nabla u_{\varepsilon \delta}\right|^{2} \psi d x d t \\
& +\iint_{X_{T}}\left(u_{\varepsilon \delta}-\theta\right)|x|^{\alpha}\left(\left|\nabla u_{\varepsilon \delta}\right|^{2}+\varepsilon\right)^{\frac{p-2}{2}} \nabla u_{\varepsilon \delta} \nabla \psi d x d t \\
& -\int_{0}^{T} \int_{\partial \Omega} g\left(\psi\left(u_{\varepsilon \delta}-\theta\right)\right) d \sigma d t=\iint_{X_{T}} f\left(\psi\left(u_{\varepsilon \delta}-\theta\right)\right) d x d t .
\end{aligned}
$$

So we have

$$
\begin{aligned}
& \iint_{X_{T}}|x|^{\alpha}\left(\left|\nabla u_{\varepsilon \delta}\right|^{2}+\varepsilon\right)^{\frac{p-2}{2}}\left|\nabla u_{\varepsilon \delta}\right|^{2} \psi d x d t \\
= & \iint_{X_{T}} f\left(\psi\left(u_{\varepsilon \delta}-\theta\right)\right) d x d t+\int_{0}^{T} \int_{\partial \Omega} g\left(\psi\left(u_{\varepsilon \delta}-\theta\right)\right) d \sigma d t \\
& +\frac{1}{2} \iint_{X_{T}} \frac{\partial \psi}{\partial t}\left(u_{\varepsilon \delta}-\theta\right)^{2} d x d t \\
& -\iint_{X_{T}}\left(u_{\varepsilon \delta}-\theta\right)|x|^{\alpha}\left(\left|\nabla u_{\varepsilon \delta}\right|^{2}+\varepsilon\right)^{\frac{p-2}{2}} \nabla u_{\varepsilon \delta} \nabla \psi d x d t .
\end{aligned}
$$

Noticing that for any $|x|^{\alpha}|\nabla v|^{p} \in L^{1}\left(X_{T}\right)$,

$$
\iint_{X_{T}} \psi|x|^{\alpha}\left(\left(\left|\nabla u_{\varepsilon \delta}\right|^{2}+\varepsilon\right)^{\frac{p-2}{2}} \nabla u_{\varepsilon \delta}-\left(|\nabla v|^{2}+\varepsilon\right)^{\frac{p-2}{2}} \nabla v\right)\left(\nabla u_{\varepsilon \delta}-\nabla v\right) d x d t \geq 0 .
$$

Falling back to (4.18), we arrive at

$$
\iint_{X_{T}} f\left(\psi\left(u_{\varepsilon \delta}-\theta\right)\right) d x d t+\int_{0}^{T} \int_{\partial \Omega} g\left(\psi\left(u_{\varepsilon \delta}-\theta\right)\right) d \sigma d t
$$




$$
\begin{aligned}
& +\frac{1}{2} \iint_{X_{T}} \frac{\partial \psi}{\partial t}\left(u_{\varepsilon \delta}-\theta\right)^{2} d x d t \\
& -\iint_{X_{T}}\left(u_{\varepsilon \delta}-\theta\right)|x|^{\alpha}\left(\left|\nabla u_{\varepsilon \delta}\right|^{2}+\varepsilon\right)^{\frac{p-2}{2}} \nabla u_{\varepsilon \delta} \nabla \psi d x d t \\
& -\iint_{X_{T}} \psi|x|^{\alpha}\left(\left|\nabla u_{\varepsilon \delta}\right|^{2}+\varepsilon\right)^{\frac{p-2}{2}} \nabla u_{\varepsilon \delta} \nabla v d x d t \\
& -\iint_{X_{T}} \psi|x|^{\alpha}\left(|\nabla v|^{2}+\varepsilon\right)^{\frac{p-2}{2}} \nabla v\left(\nabla u_{\varepsilon \delta}-\nabla v\right) d x d t \geq 0 .
\end{aligned}
$$

Similar to (3.18), we obtain that

$$
\iint_{X_{T}} \psi|x|^{\alpha}\left(|\nabla v|^{2}+\varepsilon\right)^{\frac{p-2}{2}} \nabla v\left(\nabla u_{\varepsilon \delta}-\nabla v\right) d x d t \rightarrow \iint_{X_{T}} \psi|x|^{\alpha}|\nabla v|^{p-2} \nabla v\left(\nabla u_{\delta}-\nabla v\right) d x d t .
$$

Letting $\varepsilon \rightarrow 0^{+}$, then

$$
\begin{aligned}
& \iint_{X_{T}} f\left(\psi\left(u_{\delta}-\theta\right)\right) d x d t+\int_{0}^{T} \int_{\partial \Omega} g\left(\psi\left(u_{\delta}-\theta\right)\right) d \sigma d t \\
& +\frac{1}{2} \iint_{X_{T}} \frac{\partial \psi}{\partial t}\left(u_{\delta}-\theta\right)^{2} d x d t \\
& -\iint_{X_{T}}\left(u_{\delta}-\theta\right) \vec{\zeta} \nabla \psi d x d t-\iint_{X_{T}} \psi \vec{\zeta} \nabla v d x d t \\
& -\iint_{X_{T}} \psi|x|^{\alpha}|\nabla v|^{p-2} \nabla v\left(\nabla u_{\delta}-\nabla v\right) d x d t \geq 0 .
\end{aligned}
$$

Taking $\varphi=\psi\left(u_{\delta}-\theta\right)$ in (4.16), it follows that

$$
\begin{aligned}
& \iint_{X_{T}}\left(\frac{\partial u_{\delta}}{\partial t} \psi\left(u_{\delta}-\theta\right)+\vec{\zeta} \nabla \psi\left(u_{\delta}-\theta\right)+\vec{\zeta} \psi \nabla u_{\delta}\right) d x d t \\
= & \int_{0}^{T} \int_{\partial \Omega} g \psi\left(u_{\delta}-\theta\right) d \sigma d t+\iint_{X_{T}} f \psi\left(u_{\delta}-\theta\right) d x d t .
\end{aligned}
$$

Therefore,

$$
\begin{aligned}
& \iint_{X_{T}} \vec{\zeta} \nabla \psi\left(u_{\delta}-\theta\right) d x d t \\
= & \iint_{X_{T}} f \psi\left(u_{\delta}-\theta\right) d x d t+\frac{1}{2} \iint_{X_{T}} \frac{\partial \psi}{\partial t}\left(u_{\delta}-\theta\right)^{2} d x d t \\
& -\iint_{X_{T}} \vec{\zeta} \psi \nabla u_{\delta} d x d t+\int_{0}^{T} \int_{\partial \Omega} g \psi\left(u_{\delta}-\theta\right) d \sigma d t .
\end{aligned}
$$

Substituting the above equation into (4.19) yields

$$
\begin{aligned}
& \iint_{X_{T}} f \psi\left(u_{\delta}-\theta\right) d x d t+\frac{1}{2} \iint_{X_{T}} \frac{\partial \psi}{\partial t}\left(u_{\delta}-\theta\right)^{2} d x d t \\
& +\int_{0}^{T} \int_{\partial \Omega} g \psi\left(u_{\delta}-\theta\right) d \sigma d t-\int_{0}^{T} \int_{\partial \Omega} g \psi\left(u_{\delta}-\theta\right) d \sigma d t \\
& -\iint_{X_{T}} f \psi\left(u_{\delta}-\theta\right) d x d t-\frac{1}{2} \iint_{X_{T}} \frac{\partial \psi}{\partial t}\left(u_{\delta}-\theta\right)^{2} d x d t
\end{aligned}
$$




$$
\begin{aligned}
& +\iint_{X_{T}} \vec{\zeta} \nabla u_{\delta} \psi d x d t-\iint_{X_{T}} \psi \vec{\zeta} \nabla v d x d t \\
& -\iint_{X_{T}} \psi|x|^{\alpha}|\nabla v|^{p-2} \nabla v\left(\nabla u_{\delta}-\nabla v\right) d x d t \geq 0 .
\end{aligned}
$$

Hence

$$
\iint_{X_{T}} \psi\left(\vec{\zeta}-|x|^{\alpha}|\nabla v|^{p-2} \nabla v\right)\left(\nabla u_{\delta}-\nabla v\right) d x d t \geq 0
$$

Choosing $v=u_{\delta}-\lambda \varphi(\lambda>0)$ in the above equation, we have

$$
\iint_{X_{T}} \psi\left(\vec{\zeta}-|x|^{\alpha}\left|\nabla\left(u_{\delta}-\lambda \varphi\right)\right|^{p-2} \nabla\left(u_{\delta}-\lambda \varphi\right)\right)\left(\nabla u_{\delta}-\nabla\left(u_{\delta}-\lambda \varphi\right)\right) d x d t \geq 0
$$

that is,

$$
\iint_{X_{T}} \psi\left(\vec{\zeta}-|x|^{\alpha}\left|\nabla\left(u_{\delta}-\lambda \varphi\right)\right|^{p-2} \nabla\left(u_{\delta}-\lambda \varphi\right)\right) \nabla \varphi d x d t \geq 0
$$

Letting $\lambda \rightarrow 0^{+}$,

$$
\iint_{X_{T}} \psi\left(\vec{\zeta}-|x|^{\alpha}\left|\nabla u_{\delta}\right|^{p-2} \nabla u_{\delta}\right) \nabla \varphi d x d t \geq 0
$$

follows. Similarly, if we choose $\lambda<0$, then we obtain the inverse inequality. Therefore,

$$
\iint_{X_{T}} \psi\left(\vec{\zeta}-|x|^{\alpha}\left|\nabla u_{\delta}\right|^{p-2} \nabla u_{\delta}\right) \nabla \varphi d x d t=0 .
$$

Note that $\psi=1$ on $\operatorname{supp} \varphi$, which implies (4.17) holds.

In addition, we also have (4.4) and (4.5). In fact,

$$
\begin{aligned}
\int_{F}\left|u_{\delta}(x, t)-u_{\delta 0}\right|^{2} d x & \leq 3 \int_{F}\left|u_{\delta}(x, t)-u_{\varepsilon \delta}(x, t)\right|^{2} d x \\
& +3 \int_{F}\left|u_{\varepsilon \delta}(x, t)-u_{\varepsilon \delta 0}\right|^{2} d x+3 \int_{F}\left|u_{\varepsilon \delta 0}-u_{\delta 0}\right|^{2} d x .
\end{aligned}
$$

For simplicity, we denote the three terms of the right-hand sides above by $I_{1}, I_{2}, I_{3}$. It is clear that $I_{2}, I_{3} \rightarrow 0$ as $t \rightarrow 0$ and $\varepsilon \rightarrow 0$ uniformly. So, it remains to consider $I_{1}$. For a fixed $\delta>0$ which is appropriately small, take a smooth function $\eta \geq 0$ with $\eta(t)=1$ for $t \in(0, T-2 \delta), \eta=0$ for $t \in(T-\delta, T]$ and $\left|\eta^{\prime}(t)\right| \leq \frac{C}{\delta}$. Then we have

$$
\begin{aligned}
I_{1}= & 3 \int_{t}^{T} \frac{d}{d s}\left(\int_{F} \eta(s)\left|u_{\delta}(x, s)-u_{\varepsilon \delta}(x, s)\right|^{2} d x\right) d s \\
= & 3 \int_{t}^{T}\left(\int_{F} \eta^{\prime}(s)\left|u_{\delta}(x, s)-u_{\varepsilon \delta}(x, s)\right|^{2} d x\right) d s \\
& +6 \int_{t}^{T} \int_{F} \eta(s)\left(u_{\delta}(x, s)-u_{\varepsilon \delta}(x, s)\right)\left(u_{\delta}(x, s)-u_{\varepsilon \delta}(x, s)\right)_{s} d x d s \\
\leq & \frac{C}{\delta} \int_{t}^{T}\left(\int_{F}\left|u_{\delta}(x, s)-u_{\varepsilon \delta}(x, s)\right|^{2} d x\right) d s \\
& +6\left(\int_{0}^{T} \int_{F} \eta(s)\left|u_{\delta}(x, s)-u_{\varepsilon \delta}(x, s)\right|^{2} d x d s\right)^{1 / 2}
\end{aligned}
$$




$$
\cdot\left(\int_{0}^{T} \int_{F} \eta(s)\left|u_{t \delta}(x, s)-u_{t \varepsilon \delta}(x, s)\right|^{2} d x d s\right)^{1 / 2} \text {. }
$$

Noticing that $u_{\varepsilon \delta} \rightarrow u_{\delta}$ in $L^{2}\left(X_{T}\right)$, and $u_{t \delta}, u_{t \varepsilon \delta}$ are bounded uniformly, thus, we infer that $I_{1} \rightarrow 0$ uniformly. Therefore, we arrive at (4.5). (4.4) can also be obtained by a similar approach, but we omit it.

Finally, we prove the uniqueness of solutions. Let $w$ and $v$ be the two solutions of the problem (4.2)-(4.5). For every $\varphi \in C^{\infty}\left(\overline{X_{T}}\right)$ with $\operatorname{dist}\{\operatorname{supp} \varphi, 0\}>0$, we have

$$
\iint_{X_{T}} \varphi \frac{\partial(w-v)}{\partial t} d x d t=-\iint_{X_{T}}|x|^{\alpha}\left(|\nabla w|^{p-2} \nabla w-|\nabla v|^{p-2} \nabla v\right) \nabla \varphi d x d t
$$

By an approximating process, we take $\varphi=\chi_{[0, s]}(w-v)(s \in(0, T))$, so

$$
\begin{aligned}
& \iint_{X_{s}}(w-v) \frac{\partial(w-v)}{\partial t} d x d t \\
= & -\iint_{X_{s}}|x|^{\alpha}\left(|\nabla w|^{p-2} \nabla w-|\nabla v|^{p-2} \nabla v\right) \nabla(w-v) d x d t \leq 0,
\end{aligned}
$$

where $\chi_{[0, s]}$ is the characteristic function on $[0, s]$. Hence

$$
\begin{aligned}
& \int_{F}(w(x, s)-v(x, s))^{2} d x \\
& =\int_{F}(w(x, 0)-v(x, 0))^{2} d x+\iint_{X_{s}} \frac{\partial}{\partial t}(w-v)^{2} d x d t \\
& =2 \iint_{X_{s}}(w-v) \frac{\partial(w-v)}{\partial t} d x d t \leq 0,
\end{aligned}
$$

which implies that

$$
w(x, s)=v(x, s), \quad \text { a.e. }(x, s) \in X_{T} .
$$

To prove Theorem 4.2 we need to establish the comparison theorem.

Lemma 4.4. Consider the following problems, respectively:

$$
\begin{gathered}
\frac{\partial u}{\partial t}-\operatorname{div}\left(|x|^{\alpha}|\nabla u|^{p-2} \nabla u\right)=f_{1}(x, t), \quad(x, t) \in R_{T}, \\
u(x, 0)=u_{0}(x), \quad x \in \Omega \backslash\{0\}, \\
|x|^{\alpha}|\nabla u|^{p-2} \nabla u \cdot \vec{\nu}=g_{1}(x, t), \quad(x, t) \in \partial \Omega \times(0, T), \\
\lim _{x \rightarrow 0} u(x, t)=\theta, \quad t \in(0, T)
\end{gathered}
$$

and

$$
\begin{gathered}
\frac{\partial v}{\partial t}-\operatorname{div}\left(|x|^{\alpha}|\nabla v|^{p-2} \nabla v\right)=f_{2}(x, t), \quad(x, t) \in R_{T}, \\
v(x, 0)=v_{0}(x), \quad x \in \Omega \backslash\{0\}, \\
|x|^{\alpha}|\nabla v|^{p-2} \nabla v \cdot \vec{\nu}=g_{2}(x, t), \quad(x, t) \in \partial \Omega \times(0, T), \\
\lim _{x \rightarrow 0} v(x, t)=\theta, \quad t \in(0, T),
\end{gathered}
$$

where $\theta$ is a constant. If $f_{1} \leq f_{2}, g_{1} \leq g_{2}, u_{0} \leq v_{0}$, then $u \leq v$ on $R_{T}$. Here $u$ and $v$ are the solutions of the problem (4.21)-4.24) and (4.25)-4.28), respectively. 
Proof. If $u$ and $v$ are the solutions of the problem (4.21)-(4.24) and 4.25)-(4.28), respectively, then

$$
\iint_{Q_{T}}\left(\frac{\partial u}{\partial t} \varphi+|x|^{\alpha}|\nabla u|^{p-2} \nabla u \nabla \varphi\right) d x d t-\int_{0}^{T} \int_{\partial \Omega} g_{1} \varphi d \sigma d t=\iint_{Q_{T}} f_{1} \varphi d x d t
$$

and

$$
\iint_{Q_{T}}\left(\frac{\partial v}{\partial t} \varphi+|x|^{\alpha}|\nabla v|^{p-2} \nabla v \nabla \varphi\right) d x d t-\int_{0}^{T} \int_{\partial \Omega} g_{2} \varphi d \sigma d t=\iint_{Q_{T}} f_{2} \varphi d x d t
$$

for every $\varphi \in C^{\infty}\left(\overline{Q_{T}}\right)$ with $\operatorname{dist}\{\operatorname{supp} \varphi, 0\}>0$. Here $R_{T}=(\Omega \backslash\{0\}) \times(0, T)$. Subtracting (4.30) from (4.29), and by an approximating process, we take $\varphi=H_{\eta}(u-v)$, where

$$
H_{\eta}(s)=\frac{s_{+}}{\sqrt{s^{2}+\eta}}, \quad h_{\eta}(s)=H_{\eta}^{\prime}(s), \quad \Theta_{\eta}(s)=\int_{0}^{s} H_{\eta}(\sigma) d \sigma
$$

$s_{+}=\max \{s, 0\}$. It is obvious that

$$
\begin{gathered}
h_{\eta}(s) \geq 0, \quad 0 \leq H_{\eta}(s) \leq 1, \\
\lim _{\eta \rightarrow 0} s h_{\eta}(s)=0, \quad \lim _{\eta \rightarrow 0} H_{\eta}(s)=\operatorname{sgn} s_{+}, \quad \lim _{\eta \rightarrow 0} \Theta_{\eta}(s)=s_{+} .
\end{gathered}
$$

For all $s \in(0, T)$,

$$
\begin{aligned}
& \iint_{Q_{s}} \frac{\partial(u-v)}{\partial t} H_{\eta}(u-v) d x d t \\
& +\iint_{Q_{s}}|x|^{\alpha}\left(|\nabla u|^{p-2} \nabla u-|\nabla v|^{p-2} \nabla v\right) \nabla\left(H_{\eta}(u-v)\right) d x d t \leq 0
\end{aligned}
$$

follows, that is,

$$
\begin{aligned}
& \iint_{Q_{s}} \frac{\partial(u-v)}{\partial t} H_{\eta}(u-v) d x d t \\
& +\iint_{Q_{s}}|x|^{\alpha}\left(|\nabla u|^{p-2} \nabla u-|\nabla v|^{p-2} \nabla v\right) \nabla(u-v) h_{\eta}(u-v) d x d t \leq 0 .
\end{aligned}
$$

Since $h_{\eta}(u-v) \geq 0$ and

$$
\left(|\nabla u|^{p-2} \nabla u-|\nabla v|^{p-2} \nabla v\right)(\nabla u-\nabla v) \geq 0,
$$

then

that is,

$$
\iint_{Q_{s}} \frac{\partial(u-v)}{\partial t} H_{\eta}(u-v) d x d t \leq 0
$$

$$
\begin{aligned}
& \iint_{Q_{s}} \frac{\partial}{\partial t} \Theta_{\eta}(u-v) d x d t \\
= & \int_{\Omega} \Theta_{\eta}(u-v)(x, s) d x-\int_{\Omega} \Theta_{\eta}(u-v)(x, 0) d x \\
= & \int_{\Omega} \Theta_{\eta}(u-v)(x, s) d x \leq 0 .
\end{aligned}
$$

Letting $\eta \rightarrow 0$, we have

$$
\int_{\Omega}(u-v)_{+} d x \leq 0
$$


which implies $u \leq v$ on $R_{T}$.

Lemma 4.5. Consider the following problems, respectively:

$$
\begin{gathered}
\frac{\partial u}{\partial t}-\operatorname{div}\left(|x|^{\alpha}|\nabla u|^{p-2} \nabla u\right)=f_{1}(x, t), \quad(x, t) \in R_{T}, \\
u(x, 0)=u_{0}(x), \quad x \in \Omega \backslash\{0\}, \\
u(x, t)=g_{1}(x, t), \quad(x, t) \in \partial \Omega \times(0, T), \\
\lim _{x \rightarrow 0} u(x, t)=\theta, \quad t \in(0, T)
\end{gathered}
$$

and

$$
\begin{gathered}
\frac{\partial v}{\partial t}-\operatorname{div}\left(|x|^{\alpha}|\nabla v|^{p-2} \nabla v\right)=f_{2}(x, t), \quad(x, t) \in R_{T}, \\
v(x, 0)=v_{0}(x), \quad x \in \Omega \backslash\{0\}, \\
v(x, t)=g_{2}(x, t), \quad(x, t) \in \partial \Omega \times(0, T), \\
\lim _{x \rightarrow 0} v(x, t)=\theta, \quad t \in(0, T),
\end{gathered}
$$

where $\theta$ is a constant. If $f_{1} \leq f_{2}, g_{1} \leq g_{2}, u_{0} \leq v_{0}$, then the solutions $u$ and $v$ of the problems (4.31)-(4.34) and (4.35)-(4.38) satisfy that $u \leq v$ on $R_{T}$.

By using a similar method as in Lemma 4.4, the proof can be completed. We omit it.

Now we prove Theorem 4.2 ,

Proof of Theorem 4.2. For any $\varphi \in C^{\infty}\left(\overline{Q_{T}}\right)$, since $\operatorname{dist}\{\operatorname{supp} \varphi, 0\}>0$, choose $\delta$ such that $\delta=\frac{1}{2} \operatorname{dist}\{\operatorname{supp} \varphi, 0\}>0$ in $B_{\delta}$. By the boundedness of the $L^{p}$ norm of $\nabla u_{\delta}$, we get the boundedness of the $L^{p}$ norm of $\nabla u$. Then it is easy to verify that

$$
\iint_{Q_{T}}|x|^{\alpha}|\nabla u|^{p-2} \nabla u \nabla \varphi d x d t \leq C,
$$

where $|x|^{\alpha}>0$ and $C$ is independent of $\delta$. So utilizing the equation (4.6), we can prove that $u$ satisfies the equation (1.1) and the boundary value condition (1.3) in the sense of distribution. It is rather easy to verify (1.2), since we have known that the solution satisfies $u(x, 0)=u_{0}(x)$ locally. We only need to use the unit decompose technique to prove (1.2). Next we prove (4.1). Let $w$ and $v$ be the solutions of the general steady problems, of which the nonlinear boundary value conditions are $M_{1}$ and $M_{2}$ and the right sides of the equations are $N_{1}$ and $N_{2}$, respectively. Since

$$
\begin{aligned}
& \iint_{Q_{T}}\left(\frac{\partial w}{\partial t} \varphi+|x|^{\alpha}|\nabla w|^{p-2} \nabla w \nabla \varphi\right) d x d t \\
\leq & \iint_{Q_{T}}\left(\frac{\partial u}{\partial t} \varphi+|x|^{\alpha}|\nabla u|^{p-2} \nabla u \nabla \varphi\right) d x d t \\
\leq & \iint_{Q_{T}}\left(\frac{\partial v}{\partial t} \varphi+|x|^{\alpha}|\nabla v|^{p-2} \nabla v \nabla \varphi\right) d x d t,
\end{aligned}
$$

we select $w$ and $v$ as the sub-solution and sup-solution for the evolutionary problem. Considering the two radially symmetric steady problems, we have if $p>n+\alpha, \lim _{x \rightarrow 0} w(x)=$ $\lim _{x \rightarrow 0} v(x)=\theta$ holds. Using the comparison theorem we proved above, we arrive at (4.1). 
Finally we prove the uniqueness of solutions. Let $u$ and $v$ be the two solutions of the problem (1.1)-(1.3), (4.1). For every $\varphi \in C^{\infty}\left(\overline{Q_{T}}\right)$ with $\operatorname{dist}\{\operatorname{supp} \varphi, 0\}>0$, we have

$$
\iint_{Q_{T}} \varphi \frac{\partial(u-v)}{\partial t} d x d t=-\iint_{Q_{T}}|x|^{\alpha}\left(|\nabla u|^{p-2} \nabla u-|\nabla v|^{p-2} \nabla v\right) \nabla \varphi d x d t .
$$

When $p>n+\alpha$, by an approximating process, take $\varphi=\chi_{[0, s]}(u-v)(s \in(0, T))$. Then

$$
\begin{aligned}
& \iint_{Q_{s}}(u-v) \frac{\partial(u-v)}{\partial t} d x d t \\
= & -\iint_{Q_{s}}|x|^{\alpha}\left(|\nabla u|^{p-2} \nabla u-|\nabla v|^{p-2} \nabla v\right) \nabla(u-v) d x d t \leq 0,
\end{aligned}
$$

where $\chi_{[0, s]}$ is the characteristic function on $[0, s]$. So

$$
\begin{aligned}
& \int_{\Omega}(u(x, s)-v(x, s))^{2} d x \\
& =\int_{\Omega}(u(x, 0)-v(x, 0))^{2} d x+\iint_{Q_{s}} \frac{\partial}{\partial t}(u-v)^{2} d x d t \\
& =2 \iint_{Q_{s}}(u-v) \frac{\partial(u-v)}{\partial t} d x d t \leq 0,
\end{aligned}
$$

which implies

$$
u(x, s)=v(x, s), \quad \text { a.e. }(x, s) \in Q_{T} .
$$

4.2. Unremovable orifice. Assume

$$
M_{1}+\frac{N_{1}}{n}>0
$$

or

$$
M_{2}+\frac{N_{2}}{n}<0,
$$

where $N_{i}$ and $M_{i}, i=1,2$ are the same as before. It is obvious that if one of the conditions (4.39) and (4.40) holds, and $p>n+\alpha$, then there exists one and only one solution of the problem (1.1)-(1.3), (4.1).

ThEOREM 4.6. If $p>n+\alpha$ and (4.39) (or (4.40) hold, the origin is the unremovable orifice.

Proof. Select $w$ and $v$ are the sub-solution and sup-solution for the evolutionary problem. Here $w$ and $v$ are the solutions of the general steady problems, of which the nonlinear boundary value conditions are $M_{1}$ and $M_{2}$ and the right sides of the equations are $N_{1}$ and $N_{2}$, respectively. Since (4.39) (or (4.40) ) holds, we know that for the problem which is corresponding to the sub-solution $w$ or sup-solution $v$, the origin is the unremovable orifice. Utilizing Theorem 4.2, the condition (4.39) (or (4.40), the comparison theorem and the properties of the sub-solution and sup-solution, we have that if $p>n+\alpha$, the origin is unremovable.

Acknowledgments. The authors would like to express their deep thanks to the referees for their valuable suggestions for the revision of the manuscript. The authors also would like to thank Dr. Hongxing Zhao and Miss Yi Luo. 


\section{REFERENCES}

[1] E. Dibenedetto, U. Gianazza and V. Vespri, Intrinsic Harnack estimates for nonnegative local solutions of degenerate parabolic equations, Electron. Res. Announc. Amer. Math. Soc., 12(2006), 95-99 (electronic). MR 2237273 (2007d:35163)

[2] D. Andreucci, P. Bisegna and E. DiBenedetto, Homogenization and concentrated capacity for the heat equation with non-linear variational data in reticular almost disconnected structures and applications to visual transduction, Ann. Mat. Pura Appl. (4), 182(4)(2003), 375-407. MR2023645 (2004m:35015)

[3] Ya Zhe Chen and E. DiBenedetto, Hölder estimates of solutions of singular parabolic equations with measurable coefficients, Arch. Rational Mech. Anal., 118(3)(1992), 257-271. MR1158938 (93a:35092)

[4] Kazuo Kobayasi, A kinetic approach to comparison properties for degenerate parabolic-hyperbolic equations with boundary conditions, J. Differential Equations, 230(2)(2006), 682-701. MR2269939

[5] Tsung-fang $\mathrm{Wu}$, Three positive solutions for nonlinear elliptic equations in finite strip with hole, $J$. Math. Anal. Appl. 299(1)(2004), 285-299. MR2091289(2005h:35108)

[6] G. Dal Maso and I. V. Skrypnik, A monotonicity approach to nonlinear Dirichlet problems in perforated domains, Adv. Math. Sci. Appl., 11(2)(2001), 721-751. MR1907464(2003e:35022)

[7] A. K. Nandakumaran and M. Rajesh, Homogenization of a parabolic equation in perforated domain with Dirichlet boundary condition, Proc. Indian Acad. Sci. Math. Sci., 112(3)(2002), 425-439. MR 1921791 (2003i:35021)

[8] M. Rajesh, Convergence of some energies for the Dirichlet problem in perforated domains, Rend. Mat. Appl. (7), 21(1-4)(2001), 259-274. MR1884947(2002k:35033)

[9] Viět Hà Hoàng, Singularly perturbed Dirichlet problems in randomly perforated domains, Comm. Partial Differential Equations, 25(1-2)(2000), 355-375. MR.1737552 (2001g:35022)

[10] G. Dal Maso and I. V. Skrypnik, Asymptotic behaviour of nonlinear Dirichlet problems in perforated domains, Ann. Mat. Pura Appl., 174(4)(1998), 13-72. MR1746922(2001f:35043)

[11] J. Casado-Díaz, Homogenization of general quasi-linear Dirichlet problems with quadratic growth in perforated domains, J. Math. Pures Appl. (9), 76(5)(1997), 431-476. MR1460666 (99a:35015)

[12] A. Malusa, Asymptotic behaviour of Dirichlet problems with measure data in perforated domains, Comm. Partial Differential Equations, 21(7-8)(1996), 1177-1206. MR1399195 (97d:35041)

[13] A. El Hachimi and F. de Thelin, Infinitely many radially symmetric solutions for a quasilinear elliptic problem in a ball, J. Differential Equations, 128(1996), 78-102. MR 1392397(97i:35050)

[14] Ryuji Kajikiya, Multiple existence of non-radial solutions with group invariance for sublinear elliptic equations, J. Differential Equations, 186(2002), 299-343. MR1941101 (2004c:35129)

[15] Munemitsu Hirose and Masahito Ohta, Structure of positive radial solutions to scalar field equations with harmonic potential, J. Differential Equations, 178(2002), 519-540. MR1879836 (2002k:35089)

[16] A. Castro, S. Gadam and R. Shivaji, Branches of radial solutions for semipositone problems, J. Differential Equations, 120(1995), 30-45. MR1339668(96h:35058)

[17] Y. Naito and T. Suzuki, Radial symmetry of self-similar solutions for semilinear heat equations, $J$. Differential Equations 163(2000), 407-428. MR1758704 (2001f:35135)

[18] O. A. Ladyženskaja and N. N. Ural'ceva, Linear and quasilinear elliptic equations, English Transl., Academic Press, New York, 1968. MR0244627 (39:5941)

[19] Alois Kufner, Weighted Sobolev Spaces, John Wiley \& Sons, 1985. MR802206 (86m:46033)

[20] E. DiBenedetto, Degenerate Parabolic Equations, Springer-Verlag, New-York, 1993. MR1230384 (94h:35130) 